\title{
1 A Key Cytoskeletal Regulator of Ubiquitination Amplifies TGF $\beta$ Signaling During Mouse 2 Developmental Vascular Patterning
}

4 Ronak Shetty ${ }^{1 \#}$, Divyesh Joshi ${ }^{1 \#}$, Mamta Jain ${ }^{1}$, Madavan Vasudevan $^{3}$, Jasper Chrysolite Paul ${ }^{1}$, Ganesh

5 Bhat ${ }^{1}$, Poulomi Banerjee ${ }^{1}$, Takaya Abe ${ }^{2}$, Hiroshi Kiyonari ${ }^{2}$, K. VijayRaghavan ${ }^{4}$ and Maneesha S. Inamdar ${ }^{1,5^{*}}$

$8{ }^{1}$ Jawaharlal Nehru Centre for Advanced Scientific Research, Bangalore, India; ${ }^{2}$ RIKEN Center for Life

9 Science Technologies, Kobe, Japan; ${ }^{3}$ Bionivid, Kasturi Nagar, Bangalore, India; ${ }^{4}$ National Centre for

10 Biological Sciences, Bangalore, India; ${ }^{5}$ Institute for Stem Cell biology and Regenerative Medicine

11 (inStem), Bangalore, India.

12 \# equal contribution

$13 *$ Author for correspondence

14 Email: inamdar@jncasr.ac.in

15

16

17

18

19

20 Keywords: rudhira; BCAS3; microtubule; vascular patterning; developmental angiogenesis; TGF $\beta$

21 signaling; extracellular matrix, Smad; Smurf; ubiquitin 


\section{Abstract}

24 Vascular development involves de novo formation of a capillary plexus, which is then pruned and 25 remodeled by angiogenic events. Cytoskeletal remodeling and directional endothelial migration are essential for developmental and pathological angiogenesis. Smad-dependent TGF $\beta$ signaling controls vascular patterning and is negatively regulated by microtubules. Here we show that a positive regulator of TGF $\beta$ signaling is essential for developmental vascular patterning and microtubule stability. Rudhira/BCAS3 is known to bind microtubules and to play a nodal role in cytoskeletal remodeling and directional endothelial cell (EC) migration in vitro. We demonstrate that the molecular and cellular function of Rudhira is deployed at critical steps in vascular patterning. We generated the first floxed mice for rudhira and find that global or endothelial knockout of rudhira results in mid-gestation lethality due to aberrant embryonic and extra-embryonic vessel patterning and defective cardiac morphogenesis.

Rudhira null yolk sac ECs show random and retarded migration. Yolk sac transcriptome analysis revealed key mediators of angiogenic processes and TGF $\beta$ receptor signaling were perturbed in rudhira null mutants. Molecular and biochemical analyses showed that rudhira depletion reduced microtubule stability but increased expression of pathway inhibitors leading to high levels of SMAD2/3 ubiquitination and reduced activation. These effects were not rescued by exogenous TGF $\beta$. However, TGF $\beta$ treatment of wild type ECs increased Rudhira expression. Further, exogenous Rudhira, which promotes directional cell migration, caused increased SMAD2/3 nuclear translocation and reduced inhibitor levels. Therefore, we propose that Rudhira and TGF $\beta$ signaling are mutually dependent. Rudhira has a dual function in

42 promoting TGF $\beta$ signaling, possibly by sequestering microtubules and simultaneously preventing SMAD2/3 ubiquitination to permit EC migration and vascular patterning. TGF $\beta$ signaling and aberrant

44 human Rudhira (Breast Cancer Amplified Sequence 3, BCAS3) expression are both associated with 45 tumour metastasis. Our study identifies a cytoskeletal, cell type-specific modulator of TGF $\beta$ signaling important in development and cancer. 


\section{Author Summary}

49 Remodeling and fine patterning of the blood vasculature requires controlled and co-ordinated

50 endothelial cell (EC) migration. This is achieved by the tight regulation of complex signaling pathways in

51 developmental and adult vascular patterning. The TGF $\beta$ pathway, in particular, is important for this

52 process. Here we show that the cytoskeletal protein Rudhira plays a critical role in promoting TGF $\beta$

53 signaling in EC. We generated "knockout" mice and show that Rudhira is crucial for EC migration in

54 mouse developmental vascular patterning. In the absence of Rudhira there is increased degradation of

55 TGF $\beta$ pathway effectors resulting in reduced signaling. This affects target gene expression and

56 angiogenic processes, especially extracellular matrix remodeling and EC migration. As a result, the

57 hierarchical vascular pattern is not achieved and embryos die mid-gestation. We propose that Rudhira

58 plays a pivotal role in amplifying TGF $\beta$ signaling, which is critical for vascular remodeling in multiple

59 normal and pathological contexts.

60 


\section{Introduction}

62 Vertebrate blood vessel formation involves de novo differentiation of endothelial cells (ECs) to form a

63 primary plexus (1). This is resolved into a branched hierarchical network by EC migration and inhibition

64 of proliferation, basement membrane reconstitution and recruitment of smooth muscle cells and

65 pericytes to stabilize the network (2-4). Perturbation of the balance between pro- and anti-angiogenic

66 cues leads to endothelial activation and cytoskeletal changes resulting in sprouting, migration and

67 maturation $(5,6)$. This process is spatially and temporally orchestrated during development and adult

68 neo-angiogenesis. Recent studies have elegantly elucidated the cellular dynamics of vessel regression

69 and pruning $(7,8)$. EC migration and tube formation are key steps during angiogenesis, however,

70 molecular mechanisms that operate are incompletely defined. Random and retarded EC migration

71 results in an unpatterned, often leaky vasculature $(9,10)$.

72 A primary response of EC to angiogenic stimuli, flow induced shear stress or signals that induce pruning,

73 is the reorganization of the cytoskeleton, resulting in activation of various signaling pathways (11-13).

74 The loss of EC polarity, actin reorganization and extracellular matrix remodeling are key outcomes of

75 cytoskeletal remodeling, resulting in directional cell migration essential for angiogenesis. The

76 cytoskeleton also induces changes in cell shape and gene expression through a complex network of

77 signaling pathways by influencing transcription factor activity (14).

78 The TGF $\beta$ pathway is sensitive to cytoskeletal rearrangements and regulates EC proliferation,

79 differentiation, survival and migration (15). Multiple components of this pathway crosstalk with other

80 signaling pathways to collaboratively pattern the vasculature (16). Mouse knockouts of TGF $\beta$ pathway

81 components have demonstrated that a functional TGF $\beta$ pathway is essential for cardio-vascular

82 development. Knockout of many of the ligands, receptors, effectors or regulators results in vascular

83 developmental abnormalities and often causes embryonic death (17). In the embryonic yolk sac, 
paracrine TGF $\beta$ signaling does not affect EC specification or differentiation but regulates gene expression for assembly of robust vessels (18). Microtubules bind to and negatively regulate Smad activity (19). There is limited understanding of how cytoskeletal elements regulate the TGF $\beta$ and other signaling pathways and bring about changes in EC shape, polarity and directed migration. While several mouse mutant models of vascular patterning defects have been reported, those that perturb the cytoskeleton and associated proteins show pre-gastrulation lethality on one hand or display mild developmental phenotypes on the other $(20,21)$.

Here we investigate the role of an endothelial cytoskeletal protein Rudhira/BCAS3 in regulating developmental vascular remodeling. Rudhira is a WD40 domain-containing microtubule-binding protein expressed in vascular endothelial cells during mouse embryonic development (22). Rudhira promotes directional EC migration in vitro by rapidly re-localizing to the leading edge and mediating Cdc42 activation and filopodial extension (23).

We report that global or EC-specific rudhira deletion results in mid-gestation lethality due to aberrant vascular patterning. Knockout ECs fail to show directed migration. In rudhira mutants, hierarchical patterning of the embryonic and extra-embryonic vasculature is lost. Expectedly, this has a major effect on gene expression as seen by yolk sac transcriptome and protein expression studies. While several processes and signaling pathways involved in vascular patterning are perturbed, extracellular matrix remodeling and TGF $\beta$ signaling are majorly affected. We show that Rudhira does not induce, but promotes TGF $\beta$ signaling by suppressing expression of the inhibitor smurf2, thereby preventing SMAD2/3 ubiquitination. Further, Rudhira shows increased association with microtubules upon TGF $\beta$ 104 induction. Thus Rudhira provides cytoskeletal control of several angiogenic processes by modulating 105 TGF $\beta$ signaling. This indicates that normal Rudhira function is required to aid the EC cytoskeleton in regulating gene expression and cell migration. The identification and mechanistic deciphering of genes 
bioRxiv preprint doi: https://doi.org/10.1101/055129; this version posted May 24, 2016. The copyright holder for this preprint (which was not certified by peer review) is the author/funder. All rights reserved. No reuse allowed without permission.

107 such as rudhira, provide us a pivot on which molecular partnerships with both ubiquitous 'essential' or

108 'redundant' players can be understood and deciphered in the EC context. 


\section{Results}

\section{Endothelial rudhira is vital for embryonic development}

111 Rudhira is expressed primarily in early embryonic vascular development and neo-angiogenesis, but its

112 role in vivo is not known. Hence we generated rudhira floxed mice (Fig. 1A and S1A Fig.) and crossed

113 them to CMV-Cre for ubiquitous deletion (rudhirafloxflox; CMVCre ${ }^{+}$abbreviated to rudh $^{-/}$) or Tie-2-Cre for

114 tissue-specific ablation (rudhiraflox/flox; TekCre ${ }^{+}$abbreviated to rudh ${ }^{\mathrm{CKO}}$ ) of the rudhira locus (see Materials

115 and Methods, and S1 Fig.). While heterozygotes were viable, ubiquitous or endothelial deletion of

116 rudhira gave no live homozygous pups (S1A and S1B Tables). This indicates that endothelial deletion of

117 rudhira causes recessive embryonic lethality. Analysis of embryos from E8.5 to E11.5 showed a reduced

118 number of homozygous mutant embryos (S1A and S1B Tables) as identified by genotyping (Fig. 1B),

119 transcript (Fig. 1C) and protein (S1B and S1C Fig.) expression, suggesting that lethality occurred between

$120 \quad$ E9.0 and E11.5.

121 Since rudhira expression may be transient or undetectable in some migrating cells we also analysed the

122 effect of globally deleted rudhira $\left(\mathrm{rudh}^{-/}\right)$on development from E7.5 onwards, a stage before rudhira

123 expression is initiated (22). At E7.5 rudhira mutant embryos were indistinguishable from controls with

124 respect to morphology as well as primitive streak formation as seen by Brachyury expression (S1D Fig.).

125 E8.5 mutant embryos showed unpatterned dorsal aorta as detected by Flk1 staining (S1E Fig.). By E9.5,

126 mutant embryos were growth retarded (Fig. 1D and 2A) with defects including reduced somite number

127 (19 \pm 2 at E9.5 in mutants compared to $25 \pm 2$ in controls; $n=10)$ but expressed lineage markers of

128 ectoderm (Nestin), mesoderm (Brachyury) and endoderm (AFP) (S1F Fig.). Rudhira is known to have

129 restricted expression during vasculogenesis and primitive erythropoiesis (22). However, we did not find

130 any significant change in the expression of vascular (CD34, Flk1, Ephrin, SMA, PECAM) and early

131 hematopoietic ( $\beta$-globin, c-kit, GATA-1) markers by semi-quantitative RT-PCR (S1F Fig.) showing that the 
132 two lineages are specified in rudhira null embryos. This suggests that rudhira is not essential for lineage

133 specification and early vascular differentiation. Hence, we reasoned that the growth retardation and 134 morphological defects seen in rudhira null embryos likely arise from defects in vascular rearrangement 135 and patterning.

\section{Rudhira functions in extraembryonic vascular development}

Impaired development and embryonic lethality between E8.5-E11.5 is often the result of aberrant and

functionally impaired extra-embryonic vasculature (24). Moreover, Rudhira is strongly expressed in the yolk sac vasculature (22). Hence we analysed extraembryonic structures of mutant embryos, such as

141 yolk sac and placenta which connect the maternal and fetal vasculature. Mutant yolk sacs were pale and

142 lacked major blood vessels (Fig. 1D). Immunostaining for the blood vessel marker PECAM showed that

$143 \mathrm{rudh}^{--}$yolk sac vessels were irregular and fused, unlike the finely patterned honey-comb like vascular

144 network seen in control littermates (Fig. 1E, 1I-K). Thus mutants could form a primitive vascular plexus

145 which, however, did not undergo angiogenic remodeling. Primitive erythrocytes were dispersed all over

146 the mutant yolk sac indicating an unpatterned and leaky vasculature. Histological analyses of yolk sac

147 showed congested capillaries lined by thinner endothelium (Fig. 1F, arrowhead) as compared to controls

148 (Fig. 1F, arrow). To test whether the yolk sac vessel patterning requires endothelial rudhira or is due to 149 non-specific effects we analysed endothelial-deleted $r u d h^{\text {сKO }}$ yolk sac. Although of less severity than in 150 the global knock-out, endothelial deletion of rudhira resulted in reduced branching from major vessels, 151 vessel fusion and loss of branch hierarchy at both E10.5 and E11.5 (Fig. 1G-H and I-K). These results 152 indicate that rudhira is essential for remodeling the yolk sac vascular network. Aberrant vascular 153 remodeling in rudhira mutant embryos is likely the primary cause of death. 
Fig. 1. Rudhira is essential for vascular patterning in extraembryonic tissues. (A) Schematic showing

155 strategy for generation of floxed allele of rudhira at exon 6 . White rectangles indicate exons of the rudhira locus; black and white triangles indicate loxP and frt sequences respectively. $5^{\prime} \mathrm{P}$ and $3^{\prime} \mathrm{P}$ represent the probes that were used for Southern blot analyses. Arrows depict the positions of primers for routine genotyping of mice and embryos. (B) Genomic DNA PCR analyses showing genotype of control $(+/+)$, heterozygous knock-out (+/-) and homozygous knock-out (-/-) embryos. (C) RT-PCR analysis showing rudhira mRNA expression in control (+/+) and homozygous knock-out (-/-) embryos. GAPDH RNA was used as a loading control. (D-H) Yolk sac morphology of control and rudh-/(rudhfl/fl;CMVCre+) at E9.5 in whole mount unstained (D), immunostained for PECAM/CD31 (E) and sectioned and stained with hematoxylin-eosin (F). en: endoderm, m: mesoderm. (G, H) Yolk sac vasculature marked by PECAM staining in control and rudh ${ }^{\mathrm{CKO}}$ (rudhfl/fl;TekCre+) at E10.5 and E11.5. (IK) Graphs showing quantitation of number of secondary vessels, branch points and lumen size in control and rudh-/- (rudhfl/fl;CMVCre+) at E9.5 and control and rudh ${ }^{\mathrm{CKO}}$ (rudhfl/fl;TekCre+) at E10.5 and E11.5.

Placental circulation is vital for nourishment and development of the embryo. Improper development of the labyrinth, the feto-maternal interface, results in poor fetal invasion and causes growth retardation (25). Morphological analyses showed that rudhira null embryos have a smaller placenta (S2A, S2B and S2M Fig.) and with abnormal histology (S2A and S2B Fig.) as compared to controls of the same stage. Control placenta showed a distinct chorionic plate, labyrinth, spongiotrophoblast and decidual layers. $\mathrm{Rudh}^{-/}$placenta lacked stratified layers with a greatly reduced labyrinth and chorionic plate composed mostly of trophoblast giant cells. Fetal blood vessels could not invade into the placenta of rudh ${ }^{-1}$ and contained fewer Ly76+ fetal erythrocytes as compared to controls where maternal (arrows) and fetal (arrowheads) blood cell pockets co-existed (S2C and S2D Fig.). Upon endothelial deletion of rudhira with Tie2-Cre $\left(r u d h^{C K O}\right)$, placental thickness was reduced in mutant embryos at both E10.5 and E11.5 (S2E, 

embryos.

Taken together, these findings suggest that rudhira is essential for fetal vessel invasion into the developing placenta. Further, growth retardation in rudh ${ }^{-/}$embryos is likely a result of defective placental circulation.

\section{Rudhira plays a key role in cardiovascular development and tissue patterning}

187 Since rudh ${ }^{-}$embryos survived to E9.5 and rudh ${ }^{C K O}$ survived to E11.5, we analyzed both genotypes for embryonic development and vascular patterning (Fig. 2A). Whole mount immunostaining of rudh $^{-1}$ embryos with anti-PECAM1 antibodies showed striking defects in the morphology and vasculature of the head, heart and intersomitic vessels (ISVs) (S3 Fig.). While control embryos had a well formed vascular network comprising major vessels giving rise to intricate secondary and tertiary branches (S3 Fig.

192 arrows), rudhira mutants showed completely disorganized head vasculature with defective vessel 193 sprouting, reduced capillaries and impaired branching of intersomitic vessels (ISV) that failed to sprout 194 into fine capillaries (S3 Fig. arrowheads). Histological analysis as well as immunostaining for 195 cardiovascular markers showed that both rudh $^{-}$and rudh ${ }^{C K O}$ embryos had collapsed, smaller heart 196 chambers, reduced endocardium development and a fused atrio-ventricular canal. Dorsal aorta was 197 discontinuous with a pronounced decrease in the lumen and intersomitic vessels were improperly 198 patterned (Fig. 2B-D). The endothelial lining was disorganized in all tissues and ECs seemed to have 199 impaired or random migration and were unable to form organized vessels. Similar phenotypes were observed in rudh ${ }^{\mathrm{CKO}}$ at E10.5 and E11.5 (Fig. 2B-D). 
Fig. 2. Absence of Rudhira leads to cardiovascular defects. Control and rudh-/- embryos at E9.5 or control and rudh ${ }^{\mathrm{CKO}}$ at E10.5 and E11.5 were analyzed as indicated. (A) Unstained embryos, (B, C) Histological analysis showing comparison of heart and dorsal aorta (DA). (D) Immunostaining analysis of heart, dorsal aorta (DA) and intersomitic vessels (ISV) using myocardial marker SMA, primitive erythroid marker Ly76 and vascular markers PECAM and Flk1. Arrows indicate the normal vascular patterning and arrowheads point to irregular and discontinuous vasculature. TS: Transverse section, LS: Lateral section, V: Ventricle, At: Atrium. Nuclei are marked by DAPI (Blue). Black or white dotted lines mark the boundary of DA. (E) Graph shows quantitation of dorsal aorta width in the thoracic region. Results shown are a representative of at least three independent experiments with at least three biological replicates. Scale bar: (A) $500 \mu \mathrm{m}$; (B, C) $100 \mu \mathrm{m}$; (D) E9.5 heart: $100 \mu \mathrm{m}$, E10.5 and E11.5 heart: $200 \mu \mathrm{m}$, E9.5 DA: $20 \mu \mathrm{m}, \mathrm{E} 10.5$ and E11.5 DA: $50 \mu \mathrm{m}$, ISV: $50 \mu \mathrm{m}$.

To test whether rudhira null ECs also show slow and random migration, we cultured yolk sac ECs and tested them in a wounding assay (Fig. 3A). Rudhira null ECs also showed reduced migration rate and decrease in directed migration (Fig. 3B and $3 \mathrm{C}$ ). Taken together, our results demonstrate a key role for Rudhira in directed cell migration, essential for vascular remodeling during developmental angiogenesis. expression. (A-C) Migration tracks (A) of control and rudh ${ }^{-/}$yolk sac endothelial cells subjected to wounding assay. Quantification of the rate of migration (B) and directionality (C) compared between

221 control and rudh $^{-/}$yolk sac endothelial cells. At least 30 cells were analysed per genotype. (D) 222 Representation of differentially expressed genes in rudh ${ }^{-/}$by volcano plot. (E-H) Transcriptome analysis.

223 (E) Venn diagram showing number of genes (unique probe sets) dysregulated in embryo and yolk sac 
upon rudhira deletion. (F) Unsupervised hierarchical clustering of differentially expressed gene changes

225 in rudh ${ }^{-/}$embryo (Emb) and yolk sac (YS) at E9.5 compared to controls. Each row represents a gene.

226 Expression intensities are displayed from green (low expression) to red (high expression). Lines on the

227 left represent the similarity between genes with the most similar expression profiles clustered together

228 with the shortest branches and represented in the dendrogram to illustrate their relationship. (G)

229 Unsupervised hierarchical clustering of differentially expressed gene changes in rudh $^{-/}$yolk sac at E9.5

230 compared to controls. Each row represents a gene, and column represents the tissue. (H) Histogram

231 showing significantly enriched Gene Ontology (GO) and Pathways $(p<=0.05$ ) harboring differentially

232 expressed genes in the embryo and yolk sac upon rudhira deletion. (I) Model depicting rudhira/BCAS3

233 gene regulatory pathway. Cytoscape $\vee 8.0$ was used to visualize the network. Results shown are a

234 representative of two biological and two technical replicates for microarray and at least three

235 independent experiments with at least three biological replicates for other experiments. Statistical

236 analysis was carried out using one-way ANOVA. Error bars (in B and C) indicate mean \pm SD. ${ }^{* * *} p<0.001$.

\section{Rudhira regulates expression of the angiogenesis network}

239 Rudhira regulates actin dynamics which is known to affect nuclear transcription. We performed whole

240 transcriptome-based analysis of gene expression in rudhira knockout yolk sac and embryos at E9.5 to

241 determine its effect on vascular remodeling. While Rudhira expression is primarily in the endothelium,

242 our studies in vitro ((23) and this report) indicated that changes in Rudhira expression and localization

243 are rapid and depend on inter-cellular and cell-ECM interactions. Hence we chose to analyze intact

244 tissue with minimal manipulation to understand how Rudhira affects the transcriptome. While embryos

245 at E9.5 have a diverse set of derivatives of all three germ layers, yolk sacs are primarily made of

246 primitive endoderm and mesoderm, the latter comprising mainly endothelial and hematopoietic

247 lineages. Hence we separately analyzed rudh ${ }^{-/}$embryos and yolk sacs to avoid confounding effects of 
possible mosaic deletion of rudhira in the Tie2-Cre conditional knockout. We then extensively validated

the data by quantitative PCR-based expression analysis of yolk sac RNA and endothelial cell line RNA.

Volcano plot based method was used to visualize the transcripts that are two-fold differentially expressed in yolk sac (Fig. 3D). 3291 unique probes showed 2-fold or greater statistically significant changes in gene expression (S2 Table). Of these 546 were downregulated and 334 upregulated in embryo and 566 downregulated and 1960 upregulated in yolk sac (Fig. 3E). 29 downregulated genes and 43 upregulated ones were common between embryo and yolk sac (Fig. 3E and S3 Table). Unsupervised hierarchical cluster analysis showed that genes with similar expression patterns were clustered together with branch distance proportional to their similarity in expression pattern. A distinct sub set showed reciprocal expression between embryo and yolk sac (Fig. 3F). Interestingly the majority of clustered genes were mainly upregulated in the yolk sac (Fig. 3F and 3G), while the embryo had a more balanced distribution in each cluster (Fig. 3F). To define how changes in gene expression caused by rudhira depletion may influence vascular development and remodeling, we functionally annotated the data using DAVID (Database for Annotation, Visualization and Integrated Discovery) and found that genes linked to many biological pathways were enriched. Key deregulated biological categories were identified (Fig. 3H).

Analysis of the entire data set showed greater variation between duplicates of embryo than yolk sac, possibly because of heterogeneity in the embryonic tissue. Hence for further analysis we focused on yolk sac data as it is also the primary site of vascular remodeling and shows early Rudhira expression.

267 Significant expression changes were seen in genes that relate to a range of processes or pathways, 268 which could impact on vascular development and remodeling (Fig. 3H and S4 Fig. and S5 Table). Gene ontology analysis of the common genes identified principal biological processes affected by the loss of rudhira with a Z score above 2.5. Amongst signaling pathways, negative regulation of the transforming 271 growth factor beta (TGF $\beta$ ) signaling pathway was identified as significantly perturbed. Other pathways 
272 implicated in vascular development such as Wnt, JAK/STAT and Notch signaling showed changed levels

273 of a few genes but expression of the majority of the pathway components was unaffected. Important

274 regulators of cellular processes such as angiogenesis/blood vessel remodeling, extracellular matrix,

275 regulation of proteolysis, negative regulation of peptidase activity and cell projection organization were

276 identified. Further, key molecular families involved in cytoskeletal remodeling, cell adhesion, cell

277 migration and TGF $\beta$ and VEGF pathways (all important during angiogenesis) were connected by Rudhira/

278 BCAS3 allowing us to identify the Rudhira network in angiogenesis (Fig. 3I and S6 Table).

\section{Identification of regulatory networks and nodes regulated by Rudhira}

281 A total of 140 genes from cluster analysis were enriched in the key gene ontology (GO) and pathways

282 identified (S5 Table) with a significance criterion of $\mathrm{p}<0.05$. Further, we were able to associate GOs and

283 pathways known to co-operate during vascular development and remodeling namely adhesion,

284 angiogenesis, cytoskeleton, ECM organisation, peptidase activity and TGF $\beta$ signaling (Fig. 3I). Genes

285 differentially expressed in these six processes were subjected to unsupervised hierarchical clustering to

286 identify molecular signatures (Fig. 4A, 5A and S4 Fig.). An interaction network of significant GO terms

287 was assembled into a GO map to depict the relationship among prominent functional categories (Fig. 4B,

288 5B and S4 Fig.). Subsequent verification of expression data was carried out for key genes known to

289 mediate these processes (Fig. 4C, 5C, 5D and S4 Fig.). 51 out of the 3407 genes that showed significant

290 variation from control in the knockout yolk sac were validated by qPCR on cDNA generated from rudhira

291 knockdown and non-silencing control endothelial cell line RNA (Fig. 4C, 5C, 5D and S4 Fig.) and 70\% of

292 these (36/51) agreed with array data. Changes in expression level of selected candidates were further

293 validated using cDNA generated from fresh E9.5 yolk sac RNA (S4F Fig.). 

$\mu \mathrm{m}$ (see also S5 Fig.). ${ }^{* *} \mathrm{p}<0.01,{ }^{* * *} \mathrm{p}<0.001$.

\section{Endothelial Rudhira regulates extracellular matrix (ECM) organization}

Yolk sac transcriptome analysis revealed several processes important for angiogenesis that were

perturbed upon rudhira depletion. Extracellular matrix was an over-represented functional category, in

311 Controlled ECM remodeling is important for cell migration. Mutations that affect the dynamic regulation

312 and crosstalk between the cell and ECM are also known to affect tissue patterning and homeostasis (26).

313 MMP10, MMP21 and MMP25 were aberrantly expressed while TIMPs (Tissue inhibitors of

314 metalloproteases) were not significantly changed in rudh ${ }^{-/}$yolk sacs. Expression of a large number of the

315 serine protease inhibitors was aberrant with eight members downregulated and eleven upregulated

316 (Fig. 4B and S2 Table). Interestingly several members of the extracellular Serpina clade were 317 downregulated (6/8) whereas the intracellular Serpinb clade was upregulated (8/9). Serpinb clade 
members inhibit Granzyme (Gzm) activity (27). In the rudh ${ }^{-/}$yolk sac Gzmd and Gzmg transcripts were upregulated suggesting a loss of balance between production of proteases and protease inhibitors. A disintegrin and metallopeptidase domain (Adam) class of endopeptidases namely Adam11, Adam24, Adam32, Adam7, Adamts12, and Adamts15 were all significantly upregulated in rudh $^{-/}$yolk sacs. This suggested that matrix organization or degradation may be affected. a major component of the vascular basement membrane. Of 11 collagen family members affected by

325 loss of rudhira, 7 were downregulated (Fig. 4B). In situ zymography on live rudh $^{\text {CKO }}$ yolk sac and embryo sections at E10.5 using dye quenched (DQ) gelatin showed absence of or significant decrease in signal around vessels indicating reduced matrix degradation as compared to controls (Fig. 4D and S5A Fig.). sacs and embryos showed highly disorganized collagen around the vessels (Fig. 4E and S5B Fig.). Further, Rudhira expression in vessels of control embryos overlapped with collagen staining (S5B and S5C Fig.). showed that the organization of the matrix components collagen, fibronectin and laminin was disrupted organisation. TGF $\beta$ signaling plays a major role in regulating genes involved in ECM deposition and

335 degradation. As levels of several transcripts of the TGF $\beta$ pathway were altered in rudhira mutant yolk sac (Fig 5A, 5B and $5 \mathrm{H}$ ) we analysed this pathway further.

\section{Regulation of TGF $\beta$ signaling by Rudhira}

TGF $\beta$ pathway was one of the major over-represented categories as levels of several of the TGF $\beta$ 
essential for vascular patterning and angiogenesis. Deletion of TGF $\beta 1$ leads to delayed wound healing (30). Ablation of TGF $\beta R I$ or TGF $\beta$ RII results in embryonic lethality with severe patterning defects $(31,32)$.

Smad7 were upregulated in rudhira knockdown ECs (Fig 5D). TGF $\beta$ is produced by the yolk sac ECs and is whereas rudh ${ }^{\text {CKO }}$ yolk sacs continued to show low pSMAD2 signal in the endothelial cells (Fig. 5E, arrowheads). Similarly, rudhira knockout embryo-derived endothelial cells or rudhira knockdown endothelial cell line (SVEC KD) treated with TGF $\beta$ also failed to activate pSMAD2 as compared to controls

(Fig. 5F and Fig. 5G respectively, and S6A Fig.). This suggests that Rudhira is essential for TGF $\beta$ pathway

357 activation via pSMAD2.

Fig. 5. Rudhira depletion deregulates TGF $\beta$ signaling machinery essential for angiogenesis. (A, B) 
used to visualize the network. Edge weighted spring embedded layout was used. (C-D) Graphs show representative positive regulators (C) and negative regulators (D) of the TGF $\beta$ pathway that were validated by qRT PCR on wild type (WT), non-silencing control (NS) and rudhira knockdown (KD) endothelial cell (EC) lines (SVEC). (E-F) Untreated and TGF $\beta$ treated control and rudh ${ }^{\mathrm{CKO}}$

368 (rudhfl/fl;TekCre+) yolk sacs (E) or yolk sac derived cells (F) were analyzed for the expression of phosphorylated SMAD2 (pSMAD2) by immunostaining. Samples were co-stained with endothelial markers PECAM (E) or VCAM (F). Graphs show mean fluorescence intensity for pSMAD2. $n=3$ yolk sacs. (G) Untreated and TGF $\beta$ treated non-silencing control (NS) or rudhira knockdown (KD) SVEC lines were analyzed for expression of TGF $\beta$ pathway molecules by immunoblot of cell lysates. Graph shows pSMAD2/SMAD2 ratio. (H) Graphs showing relative migration of control (NS) and rudhira knockdown (KD) SVEC lines plated on collagen or gelatin- coated dishes and induced with TGF $\beta$. Error bars indicate

375 standard error of mean (SEM). Results shown are a representative of at least three independent experiments with at least three biological replicates. Statistical analysis was carried out using one-way ANOVA. Scale bar: (E) $20 \mu \mathrm{m} * \mathrm{p}<0.05,{ }^{* *} \mathrm{p}<0.01, * * * \mathrm{p}<0.001$. (I) KEGG TGF $\beta$ Pathway. Genes in the TGF $\beta$

378 pathway from KEGG database mapped based on fold change in rudh-/- yolk sac in comparison to control 379 to understand pathway regulation. Green: downregulated, Red: upregulated. \# indicates negative regulators and antagonists of TGF $\beta$ pathway. Blue circles indicate genes whose expression level was

381 validated in this study or processes that were found to be affected.

Rudhira functions downstream of TGF $\beta$ receptor activation

384 To test whether provision of TGF $\beta$ could rescue the cell migration defect seen upon rudhira depletion, 385 we cultured SVEC knockdown cells in the presence of exogenous TGF $\beta$. Provision of exogenous TGF $\beta$ in a cell monolayer wounding assay did not rescue the migratory defects of rudhira-depleted endothelial 
cells migrating on gelatin (Fig. $5 \mathrm{H}$ ). This suggested that the TGF $\beta$ pathway requires Rudhira for activating processes that promote cell migration. Since loss of rudhira results in an inability to activate TGF $\beta$ mediated Smad2 signaling, this could lead to an inability to migrate, resulting in angiogenic defects.

Further, rudhira depletion cell autonomously affects TGF $\beta$-dependent migration. Alternatively, since Rudhira affects multiple signaling pathways, TGF $\beta$ alone may not be sufficient to rescue the effect of rudhira deletion.

\section{Rudhira is required for microtubule stability}

395 Microtubules (MT) bind to TGF $\beta$ pathway effectors Smad2 and Smad3 thereby preventing their phosphorylation and activation (19). Binding of Smads to MT is independent of TGF $\beta$ stimulation. TGF $\beta$ triggers dissociation of the Smad complex from MT and increases Smad2 phosphorylation and nuclear translocation. Our earlier work (23) showed that Rudhira interacts with MT, which prompted us to investigate whether Rudhira is required for MT-regulated TGF $\beta$ signaling. Rudhira depletion led to reduced alpha-tubulin as well as acetylated tubulin levels in SVEC endothelial cell line as well as rudhira null primary ECs (Fig. 6A,B). MTs containing acetylated and detyrosinated tubulin are known to re- 
To test whether Rudhira alone is sufficient to activate the TGF $\beta$ pathway, we transfected endothelial cells (SVEC) with a Rudhira overexpression construct (Rudh2AGFP) and analyzed the status of TGF $\beta$

411 pathway activation. Upon ligand binding to the TGF $\beta$ receptor, Smad2/3 gets activated and translocates

412 to the nucleus $(35,36)$. Rudhira overexpression alone was not sufficient to activate TGF $\beta$ signaling and 413 cause Smad2/3 nuclear localization (Fig. 6E). However, upon addition of TGF $\beta 1$ to the culture, Rudhira 414 overexpressing cells showed markedly increased pathway activation as seen by increased nuclear

415 Smad2/3 in Rud2AGFP transfected cells compared to un-transfected or vector control cells. Further,

416 Rudhira overexpression is unable to overcome the receptor-level inhibition of TGF $\beta$ pathway using the 417 small molecule inhibitor SB431542 (SB) that binds to the Alk5 co-receptor, thereby blocking pathway 418 activation (Fig. 6E). This confirms that Rudhira can augment pathway activity but not substitute for TGF $\beta$ 419 receptor activation. It also indicates that Rudhira functions downstream of the TGF $\beta$ receptor. This is in 420 agreement with the cytoskeletal localization of Rudhira.

Fig. 6. Rudhira stabilizes microtubules and augments TGF $\beta$ pathway activation. Non-silencing control

423 (NS) or rudhira knockdown (KD) ECs were analysed for microtubule organization and stability. (A-C) 424 Expression of $\alpha$-Tubulin and acetylated-Tubulin (Ac-Tubulin) by immunoblot of cell lysates (A) and 425 localisation of acetylated-tubulin by immunostaining of fixed yolk sac cells (B) and SVEC NS and KD cells 426 (C). (D) Colocalisation analysis of Rudhira and microtubules on TGF $\beta$ induction. Graph shows 427 quantitation of Rudhira and MT colocalisation from at least 20 cells. (E) SVEC were transfected with 428 plasmid constructs for expression of GFP or Rudh2AGFP, and treated with either TGF $\beta$ or TGF $\beta+$ 429 SB431542 (TGF $\beta$ inhibitor) and analysed for SMAD2 by immunostaining. Graph shows quantitation of 430 SMAD2/3 nuclear fluorescence intensity. Compare arrows (transfected cell) and arrowheads 431 (untransfected cell). (F) qRT PCR analysis of smurf2 transcript levels in wild type (WT), GFP or 
432 Rudhira2AGFP expressing endothelial cells (SVEC). Error bars indicate standard error of mean (SEM).

433 Results shown are a representative of at least three independent experiments with at least three

434 biological replicates taken into account. Statistical analysis was carried out using one-way ANOVA. Scale

435 bar: (B-E) $20 \mu \mathrm{m} .{ }^{*} \mathrm{p}<0.05, * * \mathrm{p}<0.01, * * * \mathrm{p}<0.001$.

436

437 Interestingly, Rudhira overexpression also led to a decrease in smurf2 levels (Fig. 6F and S7C Fig.). This

438 indicates that Rudhira augments TGF $\beta$ pathway activation by increasing SMAD2/3 activation, possibly

439 due to a reduction in inhibitor levels. To test whether the converse is true, i.e. whether TGF $\beta$ affects

440 Rudhira expression, we treated wild type SVEC cultures with TGF $\beta 1$. There was a significant increase in

441 Rudhira transcript and protein levels upon TGF $\beta$ induction, as detected by qPCR and

442 immunofluorescence staining (S7A, B Fig.). This indicates that Rudhira and the TGF $\beta$ pathway are

443 mutually dependent for their expression and function.

445 Rudhira inhibits Smurf- mediated TGF $\beta$ pathway attenuation

446 Our analysis indicated that Rudhira is not an inducer but a promoter of the TGF $\beta$ signaling pathway in

447 endothelial cells that acts downstream of receptor-level activation but upstream to SMAD activation.

448 KEGG pathway analysis of the yolk sac transcriptome showed that a majority of the genes involved in

449 TGF $\beta$ signaling were deregulated upon rudhira loss. Especially, several antagonists (noggin, follistatin,

450 bambi) and negative regulators (sara, smurf1/2, smad6/7) of the pathway were upregulated (Fig. 5I).

452 Fig. 7. Rudhira inhibits Smurf- mediated inhibition of TGF $\beta$ pathway. (A) qRT PCR analysis of negative 453 regulators of TGF $\beta$ pathway in non-silencing control (NS) and rudhira knockdown (KD) endothelial cell 454 (EC) lines (SVEC) upon TGF $\beta$ treatment. (B) Untreated and TGF $\beta$ treated non-silencing control (NS) or 
rudhira knockdown (KD) SVEC lines were analysed for Smad2/3 ubiquitination by immunoprecipitation

456 (IP) of Smad2/3 and immunoblotting for ubiquitin. Cell extracts were resolved by SDS-PAGE and either

457 Coomassie- stained or immunoblotted for indicated proteins. (C) in situ Proximity ligation assay (PLA) for

458 Smad2/3 and Ubiquitin on control and rudh $^{-/}$yolk sac cultured cells with or without TGF $\beta$ treatment.

459 PLA dots represent ubiquitinated Smad2/3. Graph shows the quantitation of PLA dots per cell. (D)

460 Schematic representation of the role of Rudhira in vascular patterning. The status of TGF $\beta$ signaling and

461 microtubules in the presence (wild type) or absence (Rudhira mutant) of Rudhira is shown. Boxed insets

462 show blood vessel (red) branching and ECM (blue lining) in wild type and rudhira mutant yolk sacs. Error

463 bars $(A-C)$ indicate standard error of mean (SEM). Results shown are a representative of at least two

464 independent experiments with at least two biological replicates. Statistical analysis was carried out using one-way ANOVA. ${ }^{*} p<0.05, * * p<0.01, * * * p<0.001$

466

467 The TGF $\beta$ pathway is regulated at multiple steps by a complex combination of activators, inducers and inhibitors (37). Ubiquitin- mediated proteasomal degradation is essential for fine regulation of TGF $\beta$ 469 pathway activity in the basal as well as activated states. The E3 ubiquitin ligases for SMADs, namely 470 Smurf1 and Smurf2 (SMAD specific E3 Ubiquitin ligases) are important regulators of the pathway. They 471 control SMAD2/3 levels by ubiquitination and targeting for proteasomal degradation (38, 39). These 472 inhibitors are also targets of TGF $\beta$ pathway activation.

473 Since Rudhira depletion results in increased Smurf1/2 and inhibitory Smad (Smad6 and Smad7) levels,

474 we checked the effect of exogenous TGF $\beta$ addition on these inhibitors. While control cells show a sharp 475 decrease in the levels of these inhibitors upon addition of TGF $\beta$, Rudhira- depleted cells showed no 476 reduction in their levels, suggesting a loss of control on the negative regulators of TGF $\beta$ signaling (Fig.

477 7A). To test whether increased inhibitor transcript levels co-relate with function, we assayed for levels of 478 ubiquitinated-SMAD2/3 by immuno-pulldown of SMAD2/3 followed by immunoblotting for Ubiquitin. 
479 Rudhira depletion indeed results in increased SMAD2/3 ubiquitination, which is markedly increased on 480 TGF $\beta$ - mediated activation of the pathway (Fig. 7B). To further check the status of SMAD2/3 481 ubiquitination in vivo, we performed a Proximity Ligation Assay (PLA) for SMAD2/3 and Ubiquitin in 482 primary endothelial cell cultures derived from E9.5 control and rudh-/- yolk sacs. PLA is a robust and 483 sensitive assay to identify post-translational modifications in proteins specifically and at a single 484 molecule resolution (40). Rudhira knockout cells showed significantly increased SMAD2/3 ubiquitination 485 in vivo (marked by an increase in the number of PLA dots), both in the presence or absence of 486 exogenous TGF $\beta$ (Fig. 7C). This indicates that Rudhira promotes TGF $\beta$ pathway activation by negatively 487 regulating inhibitors and thereby checking SMAD2/3 ubiquitination.

\section{Discussion}

A balance of signal sensing and response is essential to maintain homeostasis and depends on the nature of the ECM and the responsiveness of the cytoskeleton, two very important parameters in determining cell phenotype during development and disease $(29,41,42)$. The cytoskeletal response to signals would determine whether a cell can change its shape, divide or adhere. The cytoskeleton affects nuclear stability, which impinges on chromosome scaffolds and ultimately on gene expression (43). The generic role of the cytoskeleton in cell phenotype is acknowledged and even obvious, but there are only

496 a few examples linking its organization to primary determinants of cell fate, as few tissue-specific 497 cytoskeletal components are known. Context-specific cellular responses are likely to depend on the 498 presence of such components, which use ubiquitous elements to shape context-dependent outputs. We 499 therefore chose to analyse the function of Rudhira, a cytoskeletal protein expressed predominantly in the vasculature. 
502 We report here that global or endothelial-specific deletion of rudhira resulted in mid-gestation lethality

503 with severe defects in cardiovascular patterning and tissue morphogenesis. Based on a transcriptome 504 analysis we identified key steps in blood vessel remodeling such as cell adhesion, migration and 505 modulation of extracellular matrix components that are regulated by the action of Rudhira. We show 506 the essential function of Rudhira in directing endothelial cell migration during development and 507 describe, for the first time, a regulatory network mediated by Rudhira with reference to its interacting 508 partners at both binary (regulatory) as well as physical levels (Fig. 3I). We propose a model by which 509 Rudhira/BCAS3, an endothelial cell cytoskeletal component, can regulate microtubule-mediated TGF $\beta$ 510 signaling (Fig. 7D).

512 In ECS TGF $\beta$ signaling modulates the transcriptome in a manner that would promote EC migration during 513 angiogenesis (17). Also, in the yolk sac, paracrine TGF $\beta$ - signaling regulates gene expression and ECM 514 production, deposition and remodeling, required for assembly of robust vessels (18). However, the 515 absence of Rudhira hampers TGF $\beta$ signaling, negatively affecting endothelial cell migration. Expectedly, 516 rudhira null ECs show a disorganized ECM. Matrix degradation is known to support angiogenesis in 517 multiple ways such as by physical removal of barriers to migration, release of sequestered growth 518 factors or by exposing cryptic protein sequences that function in angiogenesis $(28,44)$. MMP activity is 519 essential for releasing TGF $\beta$ for growth factor signaling, which promotes cytoskeletal remodeling seen in 520 mesenchymal migration. In the absence of Rudhira at least some of these angiogenesis promoting 521 events do not occur, resulting in a disorganized matrix, possibly not permissible for directional 522 migration. Though gelatin degradation is reduced upon rudhira depletion, expression of the cognate 523 MMPs (2, 9 etc.) is not altered. We see significant increase in MMP10, MMP21, MMP25 and reduced 524 level of MMP28 transcripts suggesting that this should cause increased degradation. However, MMPs 

Rudhira localization is cytoskeletal and is dynamic in migrating cells that change shape and adhesion

are also regulated by TGF $\beta$ signaling. The reduced matrix degradation seen in rudhira CKO yolk sacs is in concordance with reduced TGF $\beta$ signaling.

Cardiac defects are seen from early development in rudhira mutants, suggesting impaired circulation.

From E8.5 in the mouse yolk sac, blood flow dictates vessel fusion and directional cell migration resulting in vascular remodeling (45). Hence cardiac defects seen in rudhira mutants could also contribute to the vascular remodeling abnormalities and aberrant TGF $\beta$ signaling.

Rudhira overexpression in endothelial cells promotes TGF $\beta$ pathway activation. Further, addition of TGF $\beta$ induces Rudhira expression. This, in addition to other observations, indicates a positive feedback of Rudhira on TGF $\beta$ signaling and vice versa. In the absence of Rudhira this feedback is lost and it affects expression of TGF $\beta$ targets and the endothelial cell transcriptome. The upstream sequence of rudhira bears binding sites for the SMAD family of transcription factors (Smad3, Smad4), Twist subfamily of class B bHLH transcription factors, Pax (Paired box factors), Brachyury and ELK1 (member of ETS oncogene family). Further, our analysis shows that Rudhira functions after receptor activation but before or at the level of SMAD2/3 activation. This suggests that Rudhira may function to regulate transcription in response to TGF $\beta$ activation, possibly by interaction with other cytoskeletal and signaling components. properties. Microtubules regulate TGF $\beta$ signaling by binding to and preventing activation of SMADs (19).

Thus our analysis provides a platform for testing cell type-specific cross talk between TGF $\beta$ signaling and the dynamic cytoskeleton in normal development as well as pathological situations such as tumor 
547

548

549

550

551

552

553

554

555

556

557

558

559

560

561

562

563

564

565

566

567

568

569

metastasis. An increase in MT and Rudhira co-localization upon TGF $\beta$ stimulation suggests that Rudhira might serve to sequester microtubules thus inhibiting MT and Smad2/3 association and promoting SMAD2/3 phosphorylation (Fig. 6D). While targeting the interaction of MT with Smads would allow regulation of the TGF $\beta$ pathway, the ubiquitous nature of these molecules is likely to result in widespread and possibly undesirable effects of therapeutic intervention. Rudhira being restricted to the vasculature could provide a suitable tissue-specific target to regulate pathological angiogenesis and TGF $\beta$ pathway activation.

The presence of cell adhesion assemblies is dependent on the underlying matrix and on whether the surface is rigid as in 2D cell culture or embedded within a 3D ECM (46). Elucidating mechanisms by which Rudhira governs cell migration will be crucial to understanding tumor cell invasion and metastasis. Indeed the upregulation of Rudhira in metastatic tumors underscores its importance (47). Interestingly, TGF $\beta$ cross talks with several other signaling pathways and is a drug target for multiple diseases. Since TGF $\beta$ is also a key inducer of the epithelial/endothelial to mesenchymal transition, it is likely that the cytoskeletal remodeling in these transitions is mediated by Rudhira. Further studies on the role of Rudhira in normal and tumor EMT will be informative.

Given the large number of signals and their varying levels that EC encounter, it is unlikely that they respond only to one or the same concentration all across the organism. A robust response over a range of signals is likely mediated by molecules that can crosstalk with a wide variety of processes. The cytoskeleton is ideally positioned for this role. EC respond to a variety of signals resulting in a limited repertoire of cytoskeletal changes, which in turn determine cell phenotype. The presence or absence of cell type-specific components such as Rudhira could provide active decisive control of EC behaviour in 
570 response to a varying milieu of signals. Our analysis indicates that in endothelial cells Rudhira remodels

571 actin (23) and affects TGF- $\beta$ meditated gene expression (this report), which in turn results in matrix

572 degradation, making the ECM permissive to cell migration. Thus our study opens up new avenues for

573 developing strategies to regulate the vascular pattern in development and disease. This could be

574 generally applicable to various cell and tissue types and identification of new components awaits further

575 investigation.

576 


\section{Materials and Methods}

578

579

580

581

ethics

committees.

Rudhira

floxed

mice

(Accession

No.

CDB0664K: http://www2.clst.riken.jp/arg/mutant\%20mice\%20list.html) were generated as described, validated by genotyping and crossed to Cre mice to generate knockout mice (see Supporting Information and Fig. 1 and S1 Fig.).

\section{RT- PCR and qRT-PCR}

587

RNA from E9.5 embryos was isolated using TRIzol reagent (Invitrogen). Reverse transcription was performed using $2 \mu \mathrm{g}$ of DNase treated RNA and Superscript II (Invitrogen, Carlsbad, CA) according to manufacturer's instructions. Quantitative RT-PCR (qRT-PCR) was carried out using EvaGreen (BIO-RAD, CA) in Biorad-CFX96 Thermal cycler (BIO-RAD, CA). Primers used are provided in S7 Table.

\section{Immunostaining, Immunohistochemistry, Microscopy and analysis}

Embryos were dissected at desired stages between E7.5 to E11.5, fixed in $4 \%$ paraformaldehyde and processed for cryosectioning or immunostaining using standard procedures (48). Primary antibodies used were against Rudhira (23), PECAM1, CD34, Flk1, Ly76 (BD Biosciences), Brachyury (Santa Cruz

596 Biotechnology), laminin, fibronectin, Smooth Muscle Actin, acetylated tubulin, $\alpha$-tubulin (Sigma 597 Chemical Co. USA), SMAD2/3, pSMAD2 (Cell Signaling Technologies, USA). Secondary antibodies were 598 coupled to Alexa-Fluor 488 or Alexa-Fluor 568 or Alexa-Fluor 633 (Molecular Probes). Cryosections were 
stained with haematoxylin and eosin using standard protocols. Images were acquired using a stereo zoom (SZX12 Olympus) or inverted (IX70, Olympus) microscope, confocal microscopes (LSM 510 Meta and LSM 700, Zeiss) and a motorized inverted microscope with fluorescence attachment (IX81, Olympus). For details, see Supporting Information. Co-localization analysis was done using co603 localization plugin in ImageJ (NIH, USA).

\section{TGF $\beta$ induction and analysis}

Live E10.5 and E11.5 CKO yolk sacs cut into two pieces or embryo-derived cells were washed in PBS and

607 induced with $0 \mathrm{ng} / \mathrm{ml}$ (uninduced) or $10 \mathrm{ng} / \mathrm{ml}$ of TGF $\beta$ in DMEM for $2 \mathrm{~h}$ and then fixed and stained for 608 pSMAD2. At least two each of the control and CKO E10.5 yolk sacs were taken for analysis. Non-silencing control (NS) or rudhira knockdown (KD) ECs were induced with 0 or $10 \mathrm{ng} / \mathrm{ml}$ of TGF $\beta$ in DMEM for $2 \mathrm{~h}$ and analysed by immunofluorescence or western blotting for SMAD2/3 and pSMAD2. Non-silencing control (NS) or rudhira knockdown (KD) ECs were induced with 0 or $10 \mathrm{ng} / \mathrm{ml}$ of TGF $\beta$ in DMEM for $48 \mathrm{~h}$

612 and analysed by qPCR for indicated genes or immunofluorescence for SMAD2/3.

\section{Western blot analysis and immuno-precipitation}

$61550 \mu \mathrm{g}$ lysate from control or knockdown cell lines of SVEC was used for Western blot analysis by 616 standard protocols. Primary antibodies used were: SMAD2/3, pSMAD2 (Cell Signaling Technologies, 617 USA), GAPDH, acetylated tubulin, $\alpha$-tubulin (Sigma Chemical Co., USA), Ubiquitin (Clone FK2, Biomol) 618 and BCAS3 (Bethyl Labs, USA). HRP conjugated secondary antibodies against appropriate species were 619 used and signal developed by using Clarity Western ECL substrate (Biorad, USA). Western blot intensities 620 were normalised to GAPDH and quantification was carried out using ImageJ. For immuno-precipitation 
621 studies, Protein G sepharose beads coated with SMAD2/3 antibody were incubated with cell lysates for

6224 hours, clarified by centrifugation and extensively washed. Equal volumes of sample were loaded and 623 resolved by SDS polyacrylamide gel and further taken for western blot analysis.

\section{In situ PLA reaction (Duolink assay)}

626 In situ PLA reaction was performed on yolk sac primary cells. The cells were cultured, fixed, 627 permeabilised and stained with primary antibodies for SMAD2/3 and Ubiquitin as mentioned earlier.

628 Thereafter, the protocol for PLA as recommended by manufacturer (Duolink, USA) was followed. Post 629 PLA, nuclei were counterstained with DAPI.

\section{In situ zymography}

632 Unfixed embryos or yolk sacs were overlaid with tissue freezing medium, snap frozen in liquid nitrogen, 633 cryosectioned at $10 \mu \mathrm{m}$, collected onto slides and overlaid with zymography solution [20 $\mathrm{gg} / \mathrm{ml} \mathrm{DQ}$ 634 gelatin (Life Technologies, USA) in 50mMTris- $\mathrm{HCl}, 150 \mathrm{mMNaCl}, 5 \mathrm{mM} \mathrm{CaCl}_{2}$ ]. The slides were incubated 635 at $37^{\circ} \mathrm{C}$ in the dark in a moist chamber for 15 min (yolk sac) or $2 \mathrm{~h}$ (embryos), then rinsed with ultrapure 636 water (Milli-Q, Millipore) and fixed with 4\% paraformaldehyde. Multiple sections from at least four 637 embryos per genotype were analysed.

\section{Endothelial cell culture, adhesion and migration assays}


642 resuspended in culture medium (DMEM, 20\% fetal calf serum, $1 \mathrm{X}$ Glutamax, $1 \mathrm{X}$ antibiotics and $50 \mu \mathrm{g} / \mathrm{ml}$

643 Endothelial Cell Growth Supplement (ECGS) (Sigma Chemical Co., USA) and plated onto 0.1\% gelatin

644 coated dishes. Confluent monolayers were incubated with $5 \mu \mathrm{g} / \mathrm{ml}$ Dil-Ac-LDL (Invitrogen) for 4 hours to

645 mark endothelial cells, then scratched and monitored for cell migration in real time by video microscopy

646 (see Supporting Information). For assessing effects on cell migration on different substrates, SVEC plated

647 on desired matrix components were allowed to adhere and form a monolayer for $24 \mathrm{~h}$ before wounding.

648 For assaying the effect of TGF $\beta$ on migration, $5 \mathrm{ng} / \mathrm{ml} \mathrm{TGF} \beta$ was added to cultures $6 \mathrm{~h}$ before scratching

649 and continuously provided up to $12 \mathrm{~h}$ post-wounding. Wound width at $0 \mathrm{~h}$ and $12 \mathrm{~h}$ post-wounding was

650 measured and quantified as described before (23).

651

652 Microarray analysis

653 Stage-matched E9.5 embryos of control and ubiquitous knockout littermates were used for microarray 654 analysis as detailed in Supporting Information.

\section{Quantification and Statistical analyses}

Quantification and statistical analysis are described in Supporting Information.

\section{Acknowledgements}

660 We thank staff at the Jackson Laboratories, USA for inputs on mouse breeding and maintenance; 661 Developmental Studies Hybridoma Bank, University of lowa, USA for some antibodies; JNCASR Imaging 
facility, NCBS Central Imaging and Flow Facility, JNCASR Animal Facility, NCBS Animal facility for access and Inamdar laboratory members for fruitful discussions.

664

665

\section{Author Contributions}

666

667

668

669

670

671

672

673

674

675

676

677

678

679

680

681

682

683

684

685

686

687

688

689

690

691

692

693

694

M.S.I. conceived of the project and directed the work. M.S.I., R.S., D.J., J.C.P., M.J., G.B., P.B. designed and performed animal experiments, cell biology, and imaging. R.S., M.V., M.S.I., D.J. analyzed transcriptome data. M.S.I. designed floxed mice and T.A., and H.K generated floxed mice. M.S.I., R.S., M.V., K.V.R. wrote the manuscript. All authors reviewed and made comments on the manuscript.

\section{Disclosures}

Madavan Vasudevan is Co-Founder \& Director, Bionivid Technology Pvt Ltd.

\section{References}

1. Folkman J. Fundamental concepts of the angiogenic process. Curr Mol Med. 2003;3(7):643-51.

2. Davis GE, Senger DR. Endothelial extracellular matrix: biosynthesis, remodeling, and functions during vascular morphogenesis and neovessel stabilization. Circ Res. 2005;97(11):1093-107.

3. Lamalice L, Le Boeuf F, Huot J. Endothelial cell migration during angiogenesis. Circ Res. 2007;100(6):782-94.

4. van Hinsbergh VW, Engelse MA, Quax PH. Pericellular proteases in angiogenesis and vasculogenesis. Arterioscler Thromb Vasc Biol. 2006;26(4):716-28.

5. Bussolino F, Mantovani A, Persico G. Molecular mechanisms of blood vessel formation. Trends Biochem Sci. 1997;22(7):251-6.

6. Morgan JT, Pfeiffer ER, Thirkill TL, Kumar P, Peng G, Fridolfsson HN, et al. Nesprin-3 regulates endothelial cell morphology, perinuclear cytoskeletal architecture, and flow-induced polarization. Mol Biol Cell. 2011;22(22):4324-34.

7. Franco CA, Jones ML, Bernabeu MO, Geudens I, Mathivet T, Rosa A, et al. Dynamic endothelial cell rearrangements drive developmental vessel regression. PLoS Biol. 2015;13(4):e1002125.

8. Wietecha MS, Cerny WL, DiPietro LA. Mechanisms of vessel regression: toward an understanding of the resolution of angiogenesis. Curr Top Microbiol Immunol. 2013;367:3-32.

9. Jin $Y$, Jakobsson $L$. The dynamics of developmental and tumor angiogenesis-a comparison. Cancers (Basel). 2012;4(2):400-19.

10. Wang S, Aurora AB, Johnson BA, Qi X, McAnally J, Hill JA, et al. The endothelial-specific microRNA miR-126 governs vascular integrity and angiogenesis. Dev Cell. 2008;15(2):261-71. 
11. Tzima E, del Pozo MA, Shattil SJ, Chien S, Schwartz MA. Activation of integrins in endothelial cells by fluid shear stress mediates Rho-dependent cytoskeletal alignment. EMBO J. 2001;20(17):463947.

12. Wesselman JP, De Mey JG. Angiotensin and cytoskeletal proteins: role in vascular remodeling. Curr Hypertens Rep. 2002;4(1):63-70.

13. Chien S, Li S, Shiu YT, Li YS. Molecular basis of mechanical modulation of endothelial cell migration. Front Biosci. 2005;10:1985-2000.

14. Rosette C, Karin M. Cytoskeletal control of gene expression: depolymerization of microtubules activates NF-kappa B. The Journal of cell biology. 1995;128(6):1111-9.

15. Massagué J. TGF $\beta$ in cancer. Cell. 2008;134(2):215-30.

16. Guo X, Wang X-F. Signaling cross-talk between TGF- $\beta / B M P$ and other pathways. Cell research. 2009;19(1):71-88.

17. Lebrin $F$, Deckers $M$, Bertolino $P$, ten Dijke $P$. TGF- $\beta$ receptor function in the endothelium. Cardiovascular research. 2005;65(3):599-608.

18. Carvalho RL, Jonker L, Goumans MJ, Larsson J, Bouwman P, Karlsson S, et al. Defective paracrine signalling by TGFbeta in yolk sac vasculature of endoglin mutant mice: a paradigm for hereditary haemorrhagic telangiectasia. Development. 2004;131(24):6237-47.

19. Dong C, Li Z, Alvarez R, Feng X-H, Goldschmidt-Clermont PJ. Microtubule binding to Smads may regulate TGF $\beta$ activity. Molecular cell. 2000;5(1):27-34.

20. Kim GW, Li L, Gorbani M, You L, Yang XJ. Mice lacking alpha-tubulin acetyltransferase 1 are viable but display alpha-tubulin acetylation deficiency and dentate gyrus distortion. J Biol Chem. 2013;288(28):20334-50.

21. Yuba-Kubo A, Kubo A, Hata M, Tsukita S. Gene knockout analysis of two gamma-tubulin isoforms in mice. Dev Biol. 2005;282(2):361-73.

22. Siva K, Inamdar MS. Rudhira is a cytoplasmic WD40 protein expressed in mouse embryonic stem cells and during embryonic erythropoiesis. Gene Expr Patterns. 2006;6(2):225-34.

23. Jain M, Bhat GP, Vijayraghavan K, Inamdar MS. Rudhira/BCAS3 is a cytoskeletal protein that controls Cdc42 activation and directional cell migration during angiogenesis. Exp Cell Res. 2012;318(6):753-67.

24. Copp AJ. Death before birth: clues from gene knockouts and mutations. Trends in Genetics. $1995 ; 11(3): 87-93$.

25. Rinkenberger J, Werb Z. The labyrinthine placenta. Nat Genet. 2000;25(3):248-50.

26. Shah SP, Roth A, Goya R, Oloumi A, Ha G, Zhao Y, et al. The clonal and mutational evolution spectrum of primary triple-negative breast cancers. Nature. 2012;486(7403):395-9.

27. Quan LT, Caputo A, Bleackley RC, Pickup DJ, Salvesen GS. Granzyme B is inhibited by the cowpox virus serpin cytokine response modifier A. Journal of Biological Chemistry. 1995;270(18):10377-9.

28. Rundhaug JE. Matrix metalloproteinases and angiogenesis. J Cell Mol Med. 2005;9(2):267-85.

29. Lu P, Takai K, Weaver VM, Werb Z. Extracellular matrix degradation and remodeling in development and disease. Cold Spring Harb Perspect Biol. 2011;3(12).

30. Crowe MJ, Doetschman T, Greenhalgh DG. Delayed wound healing in immunodeficient TGF- $\beta 1$ knockout mice. Journal of Investigative Dermatology. 2000;115(1):3-11.

31. Larsson J, Goumans MJ, Sjöstrand LJ, van Rooijen MA, Ward D, Levéen $P$, et al. Abnormal angiogenesis but intact hematopoietic potential in TGF- $\beta$ type I receptor-deficient mice. The EMBO journal. 2001;20(7):1663-73.

32. Oshima M, Oshima $H$, Taketo MM. TGF- $\beta$ receptor type II deficiency results in defects of yolk sac hematopoiesis and vasculogenesis. Developmental biology. 1996;179(1):297-302.

33. Itoh F, Itoh S, Adachi T, Ichikawa K, Matsumura Y, Takagi T, et al. Smad2/Smad3 in endothelium is indispensable for vascular stability via S1PR1 and N-cadherin expressions. Blood. 2012;119(22):5320-8. 
34. Montagnac G, Meas-Yedid V, Irondelle M, Castro-Castro A, Franco M, Shida T, et al. [agr] TAT1 catalyses microtubule acetylation at clathrin-coated pits. Nature. 2013;502(7472):567-70.

35. Heldin $\mathrm{C}-\mathrm{H}$, Miyazono K, Ten Dijke P. TGF- $\beta$ signalling from cell membrane to nucleus through 746 SMAD proteins. Nature. 1997;390(6659):465-71.

747 36. Attisano L, Wrana JL. Mads and Smads in TGF $\beta$ signalling. Current opinion in cell biology. $748 \quad$ 1998;10(2):188-94.

749 37. Band AM, Laiho M. Crosstalk of TGF- $\beta$ and estrogen receptor signaling in breast cancer. Journal of mammary gland biology and neoplasia. 2011;16(2):109-15.

38. Lin $X$, Liang $M$, Feng $X-H$. Smurf2 is a ubiquitin E3 ligase mediating proteasome-dependent degradation of Smad2 in transforming growth factor- $\beta$ signaling. Journal of Biological Chemistry. 753 2000;275(47):36818-22.

754 39. Zhang Y, Chang C, Gehling DJ, Hemmati-Brivanlou A, Derynck R. Regulation of Smad degradation 755 and activity by Smurf2, an E3 ubiquitin ligase. Proceedings of the National Academy of Sciences. 756 2001;98(3):974-9.

757 40. Söderberg O, Gullberg M, Jarvius M, Ridderstråle K, Leuchowius K-J, Jarvius J, et al. Direct 758 observation of individual endogenous protein complexes in situ by proximity ligation. Nature methods. 759 2006;3(12):995-1000.

760 41. Cox TR, Erler JT. Remodeling and homeostasis of the extracellular matrix: implications for fibrotic diseases and cancer. Dis Model Mech. 2011;4(2):165-78.

762 42. Nelson CM, Bissell MJ. Of extracellular matrix, scaffolds, and signaling: tissue architecture regulates development, homeostasis, and cancer. Annu Rev Cell Dev Biol. 2006;22:287-309. personalities team up. Curr Opin Cell Biol. 2014;32C:39-47.

44. Stetler-Stevenson WG. Matrix metalloproteinases in angiogenesis: a moving target for therapeutic intervention. J Clin Invest. 1999;103(9):1237-41.

45. Udan RS, Vadakkan TJ, Dickinson ME. Dynamic responses of endothelial cells to changes in blood flow during vascular remodeling of the mouse yolk sac. Development. 2013;140(19):4041-50.

46. Fraley $\mathrm{SI}$, Feng $\mathrm{Y}$, Krishnamurthy R, Kim DH, Celedon A, Longmore GD, et al. A distinctive role for focal adhesion proteins in three-dimensional cell motility. Nat Cell Biol. 2010;12(6):598-604. cells and vascular precursors suggests a role in human embryogenesis and tumor angiogenesis. PLoS One. 2007;2(11):e1202.

775 48. Schlaeger TM, Qin Y, Fujiwara Y, Magram J, Sato TN. Vascular endothelial cell lineage-specific 776 promoter in transgenic mice. Development. 1995;121(4):1089-98. 

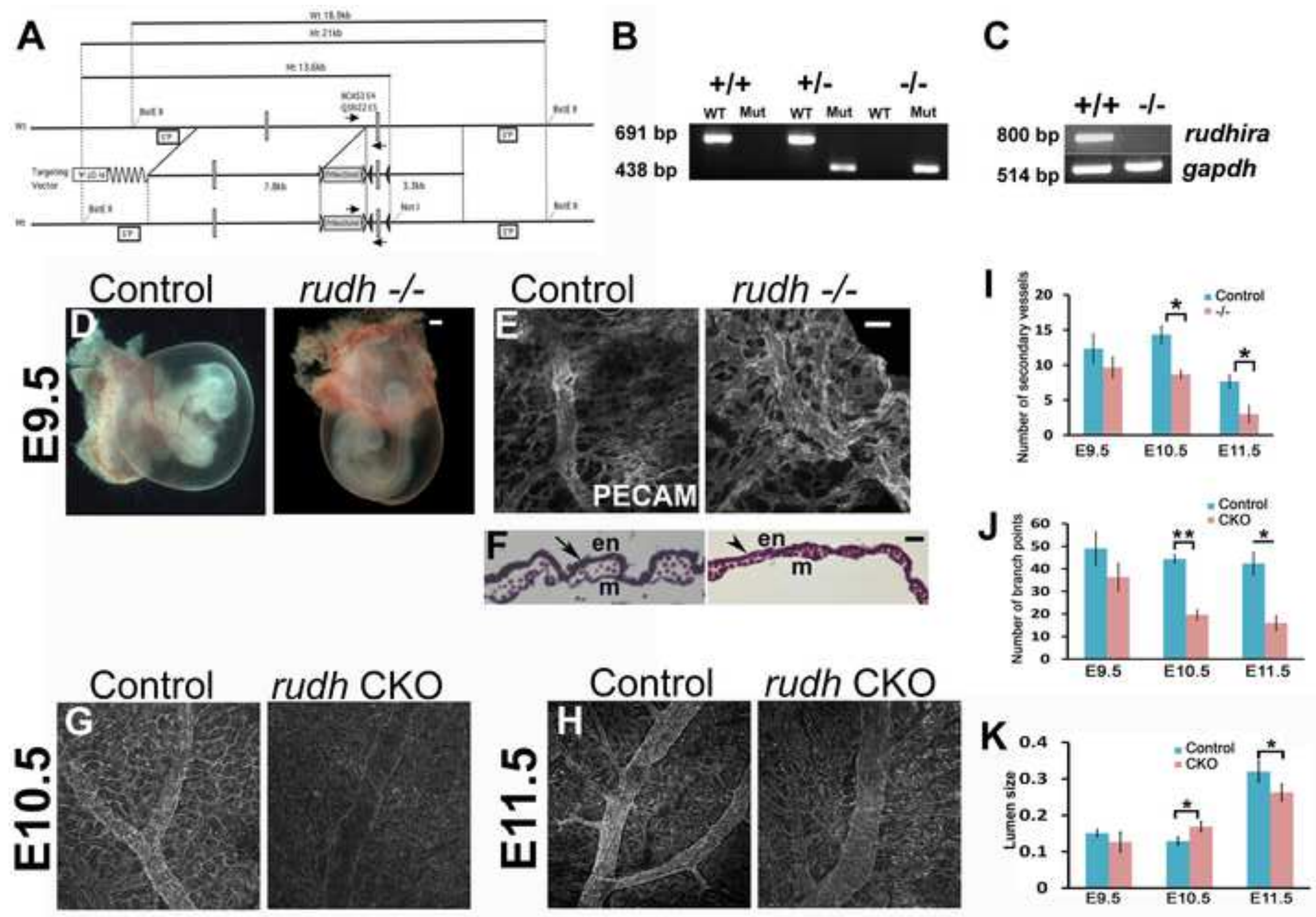

Figure 1 
E9.5

E10.5

E11.5
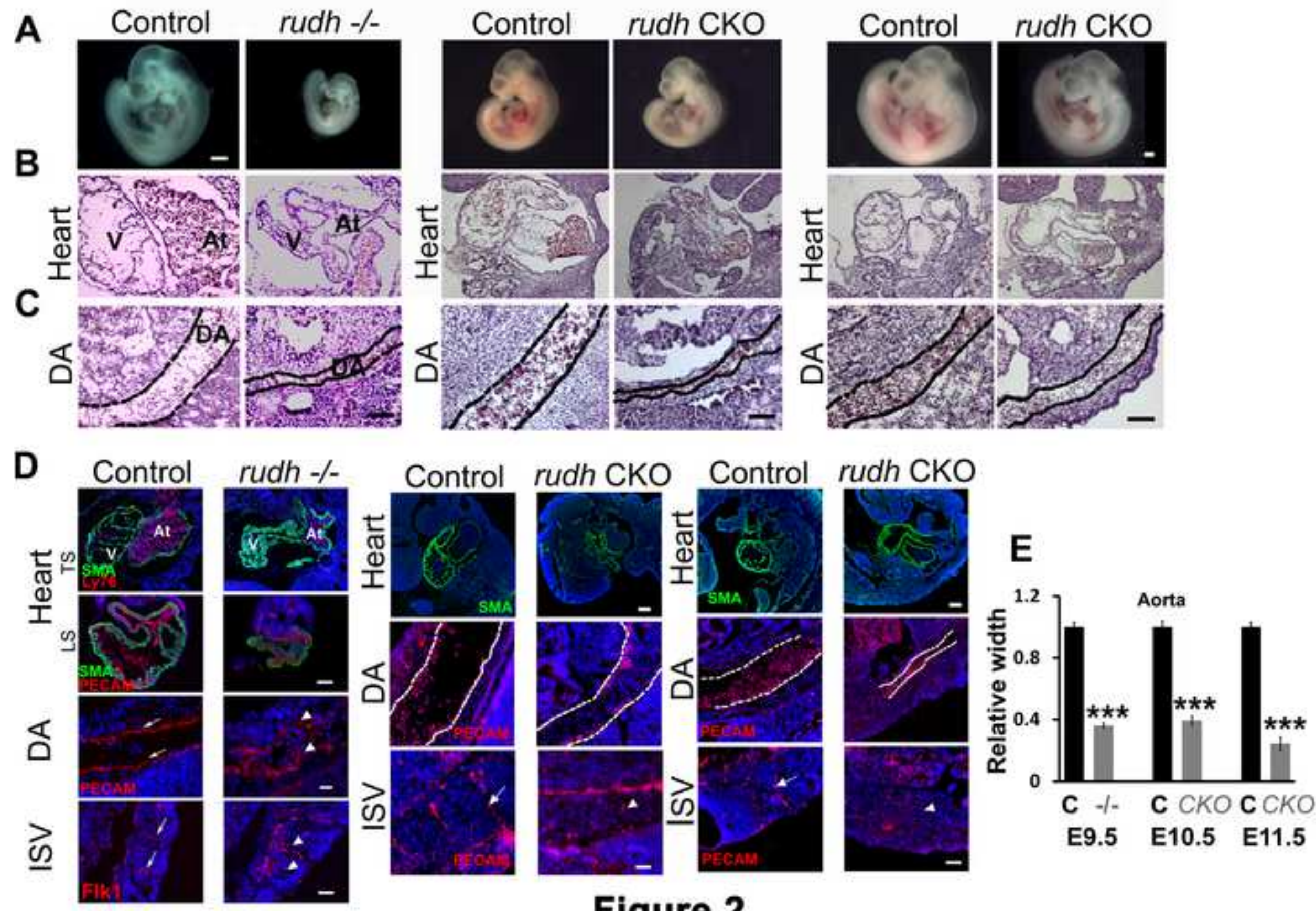

Figure 2 
A

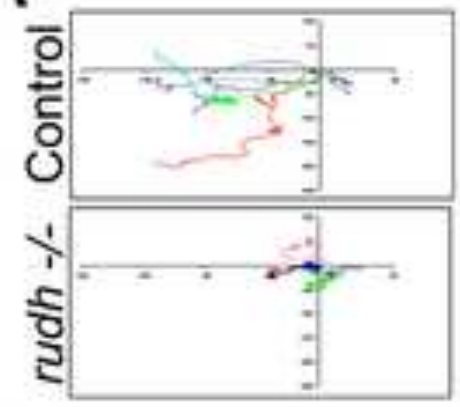

B

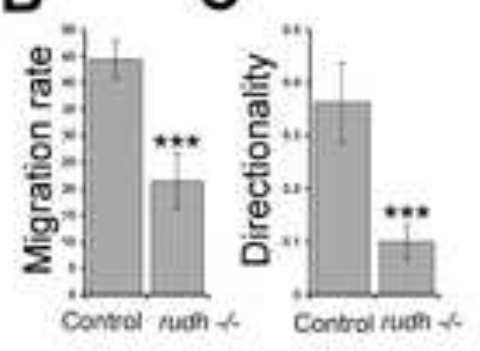

D

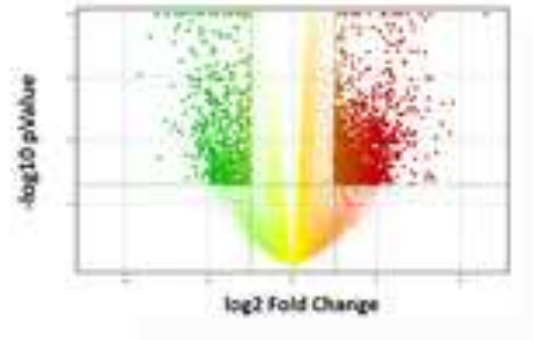

E

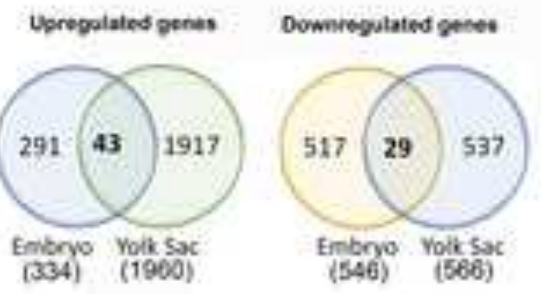

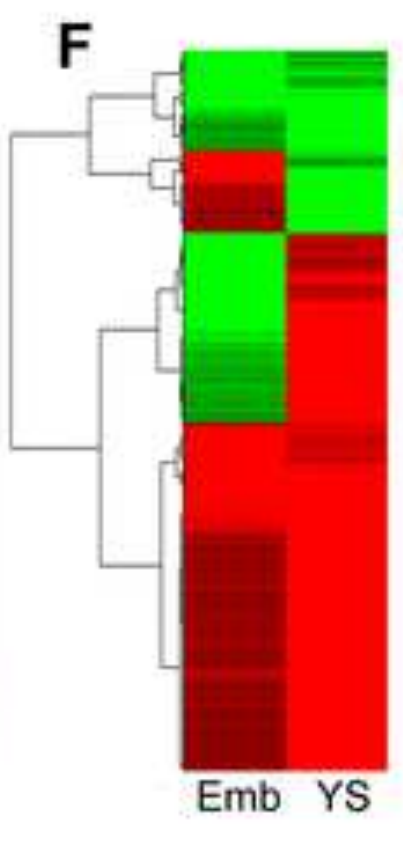

G

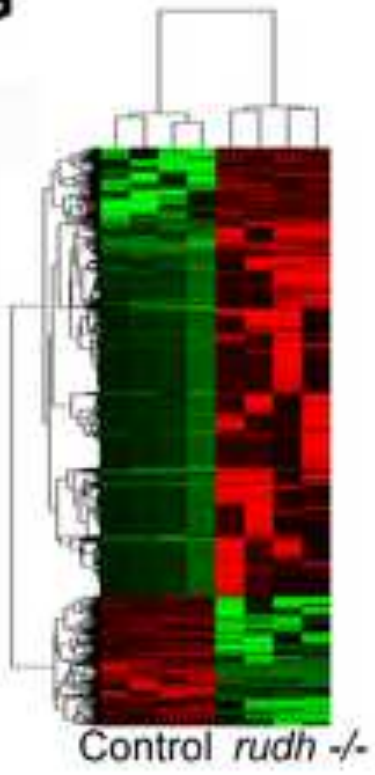

H
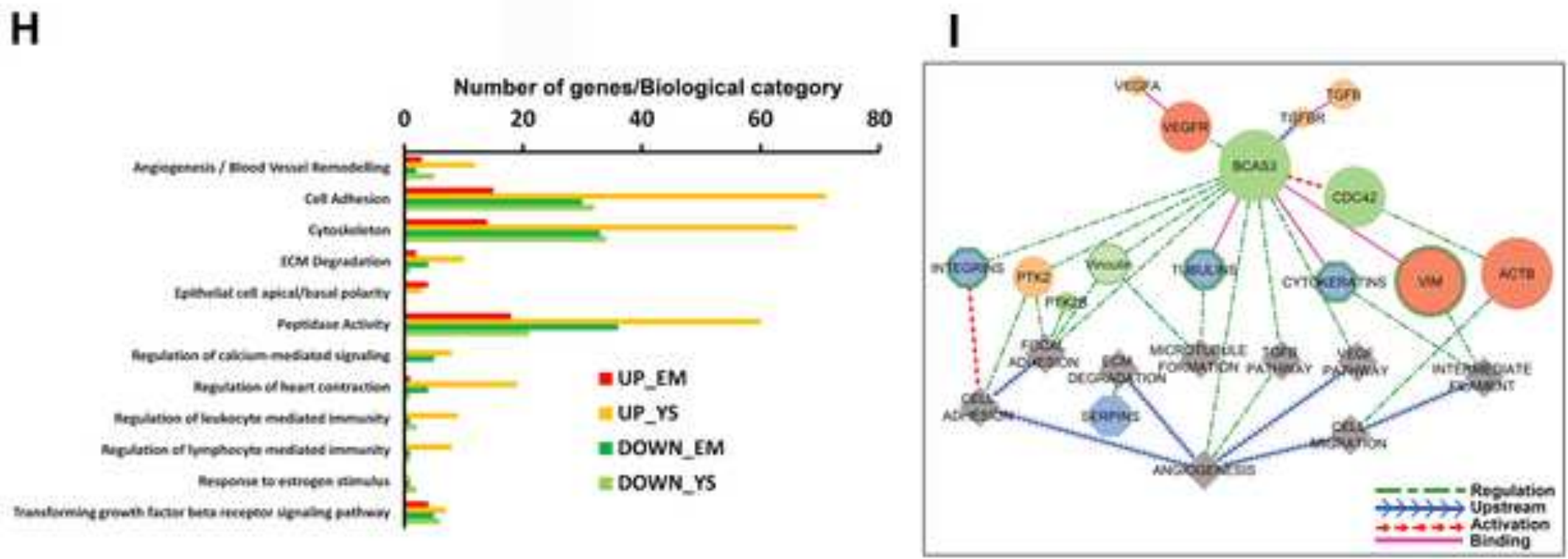

Figure 3 


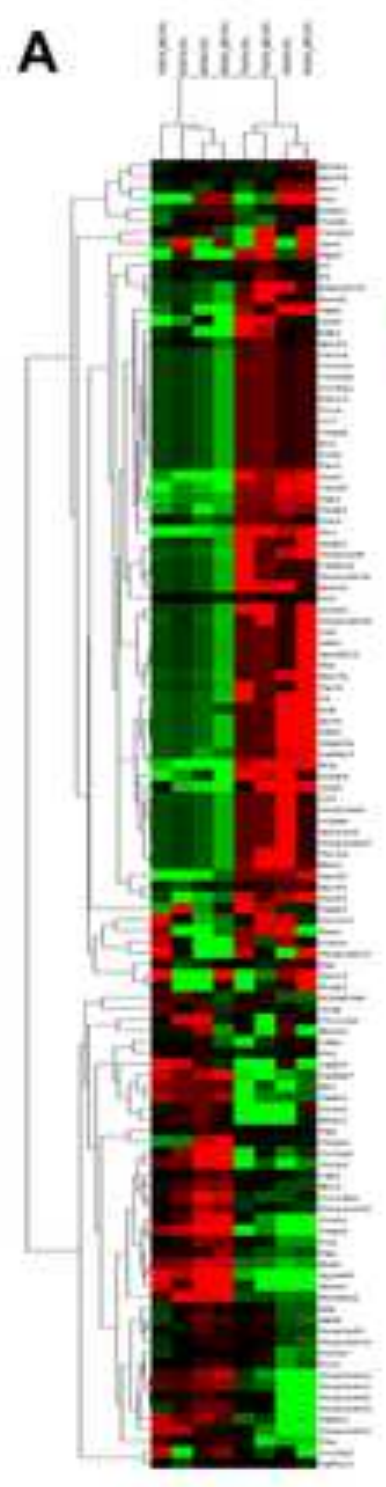

B

Extracellular matrix
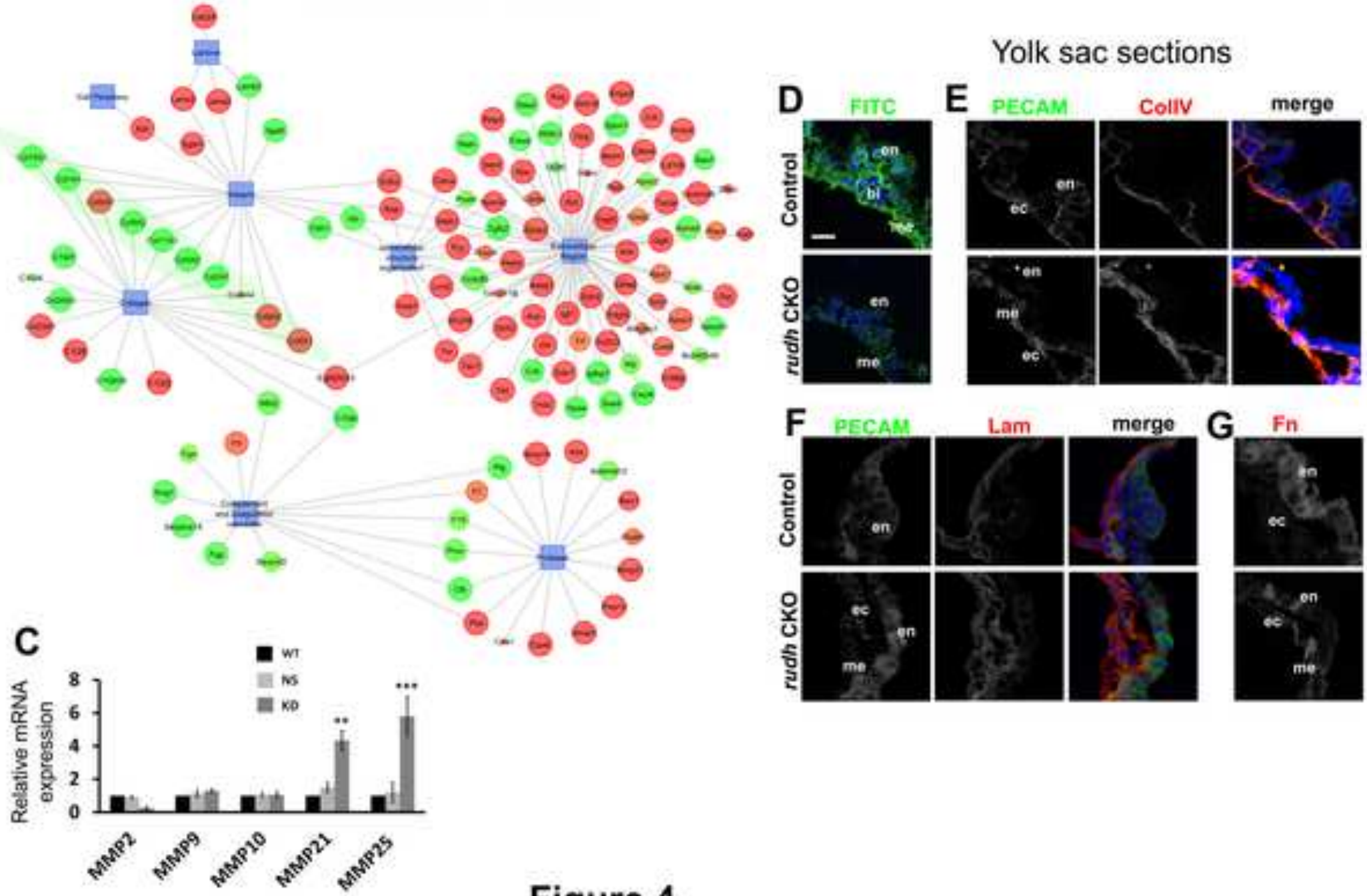

Figure 4 

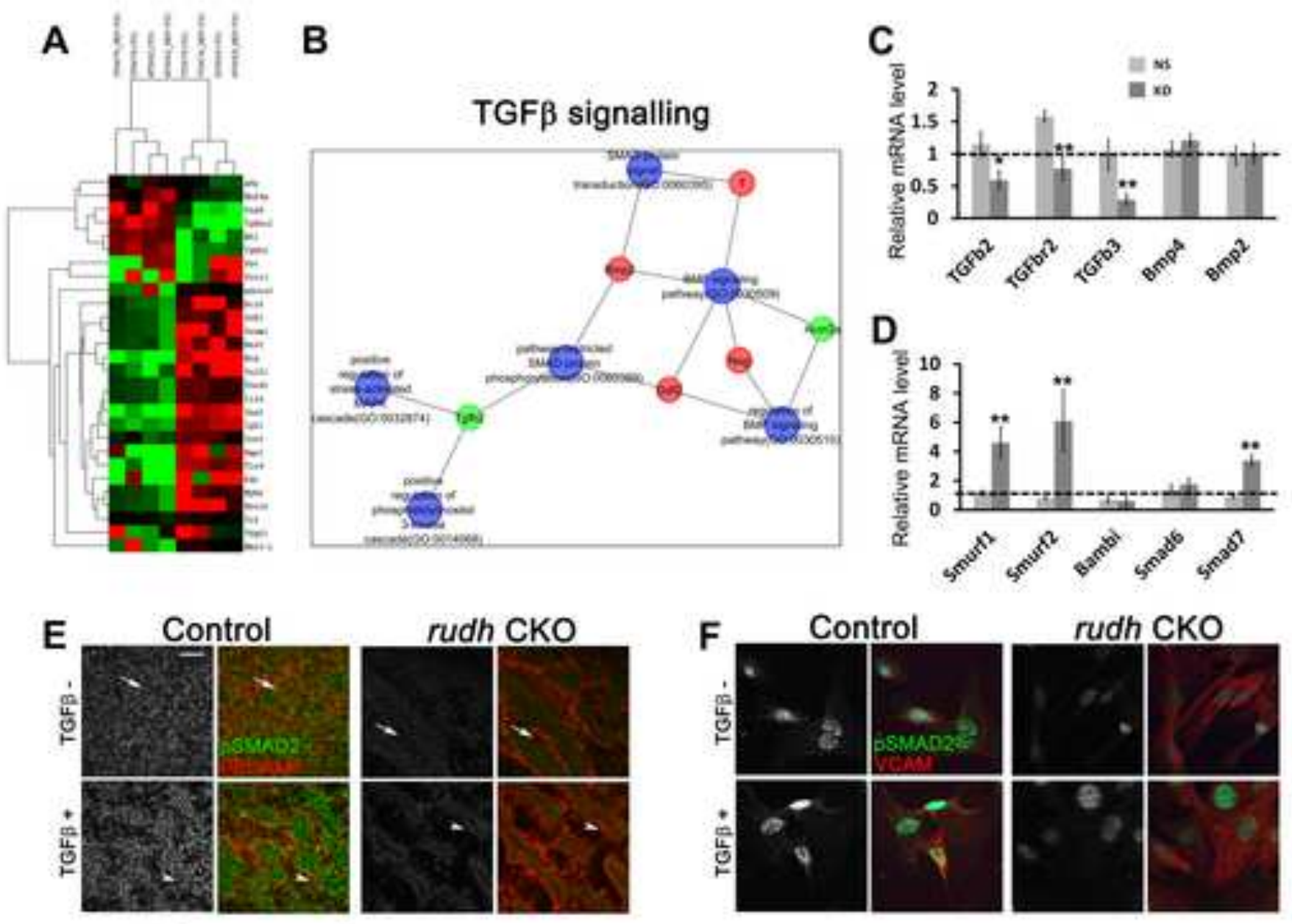

rudh CKO
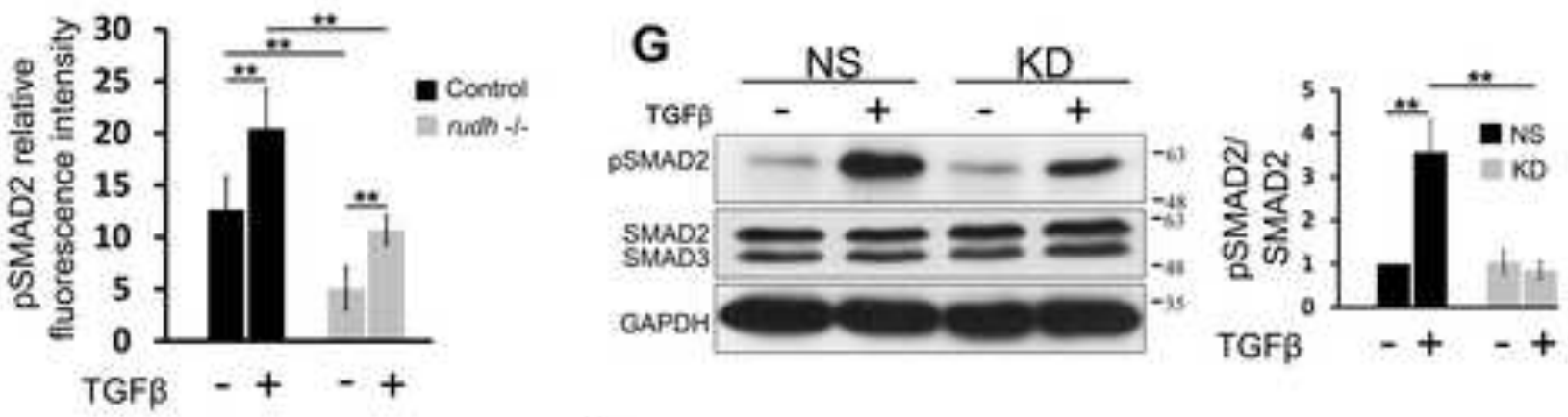

H
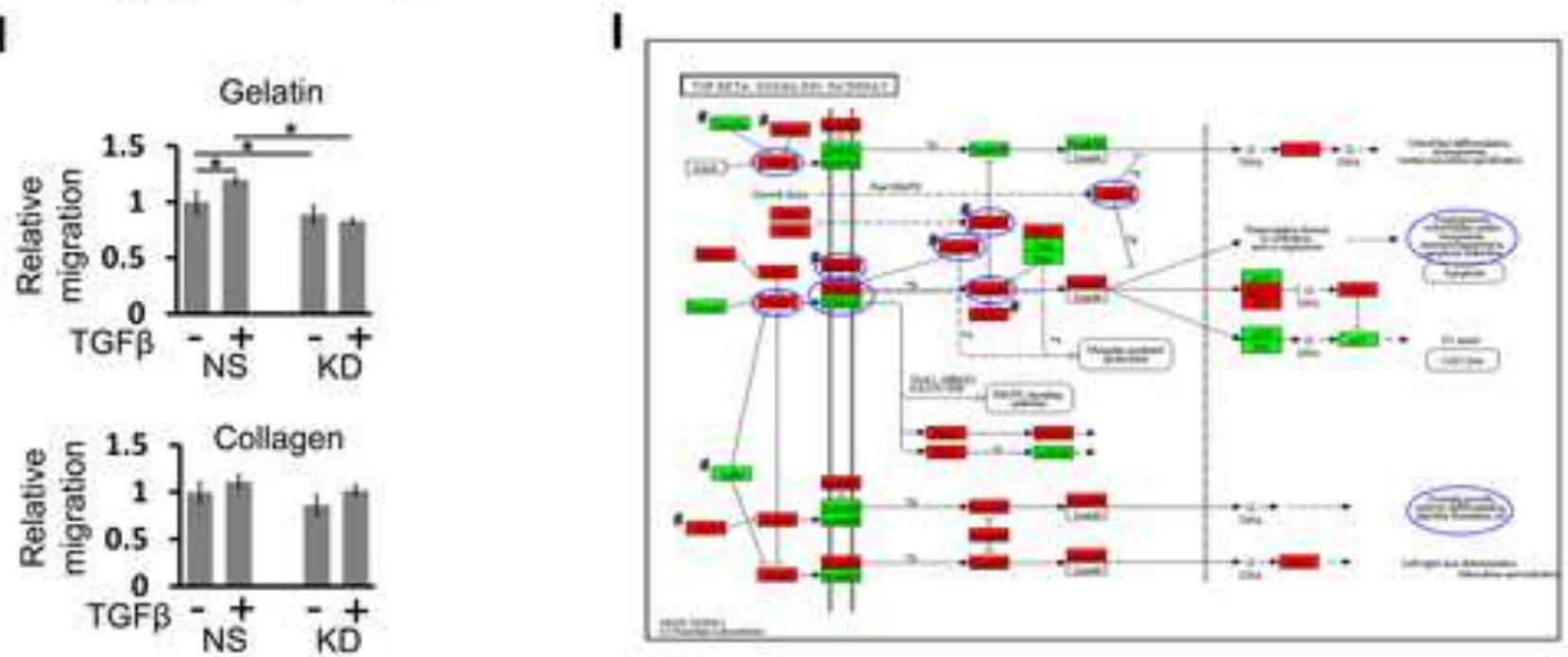

Figure 5 

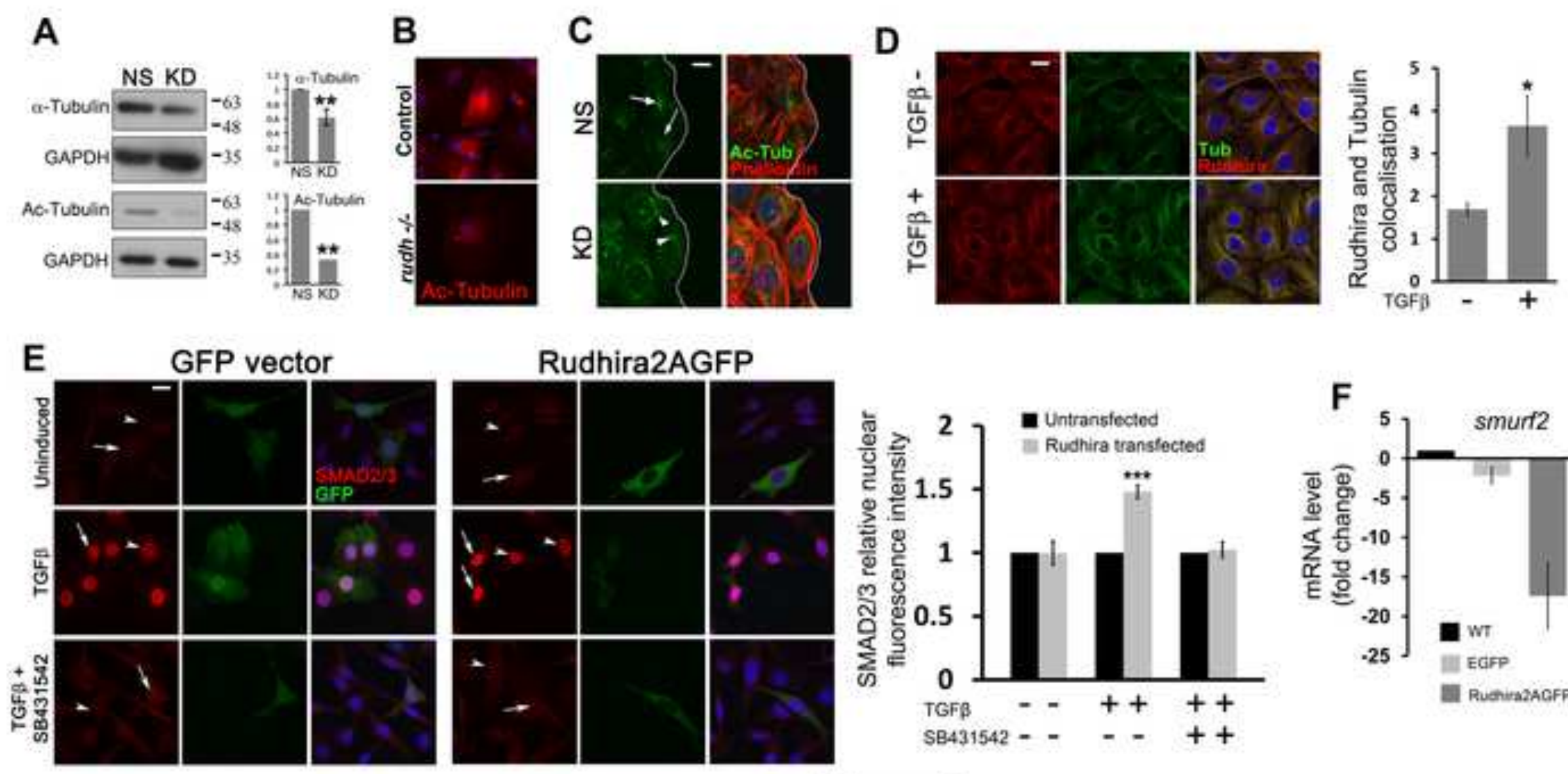

\section{Rudhira2AGFP}
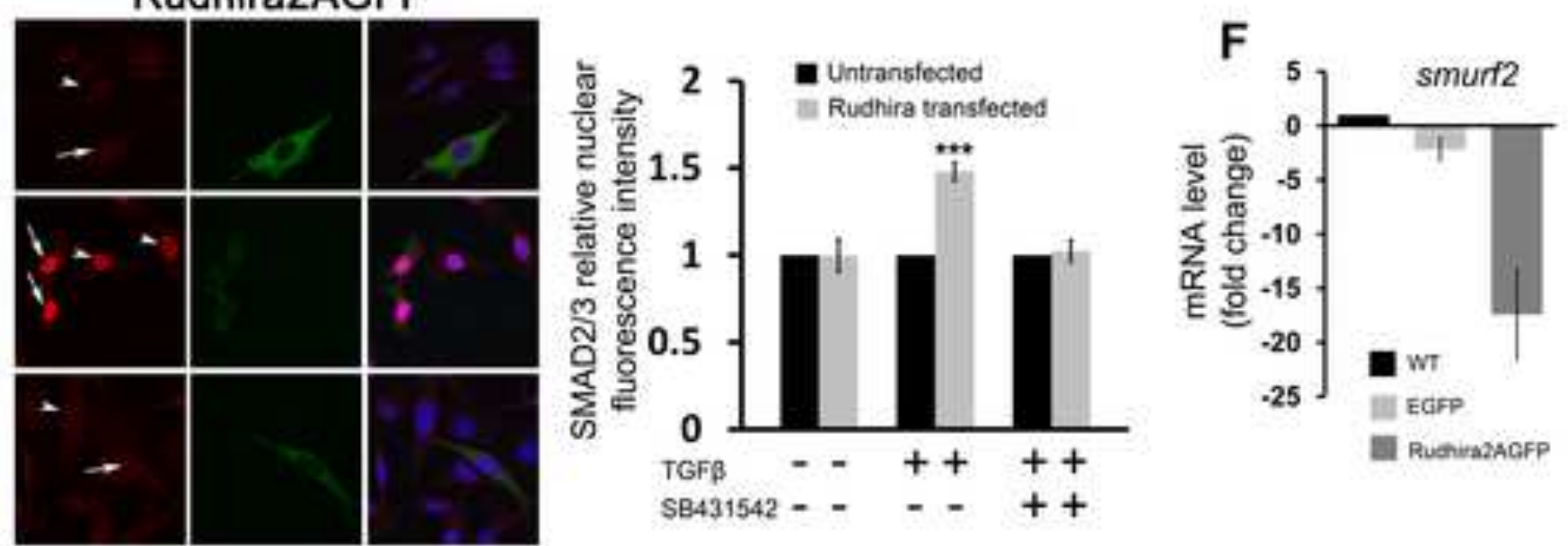

Figure 6 
A

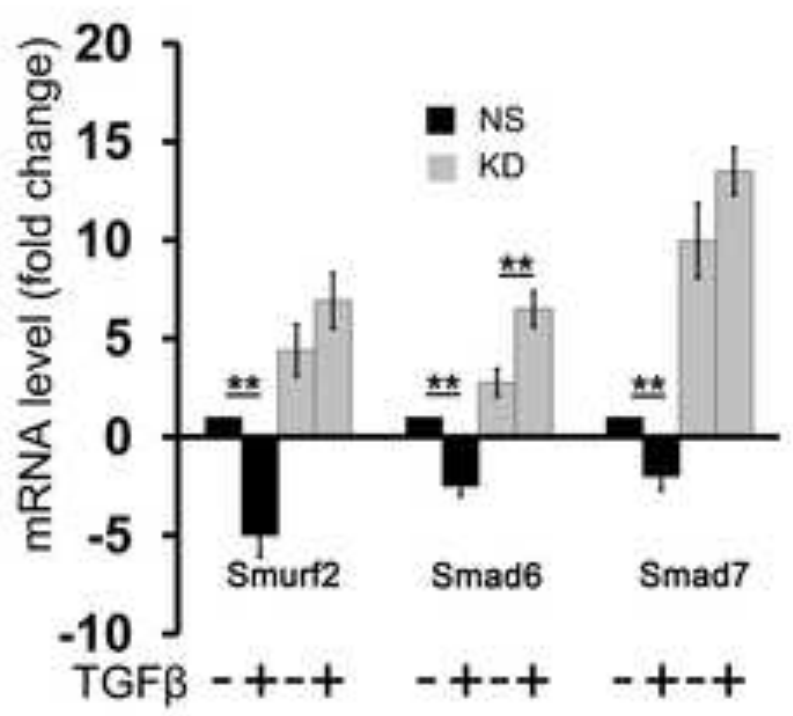

B

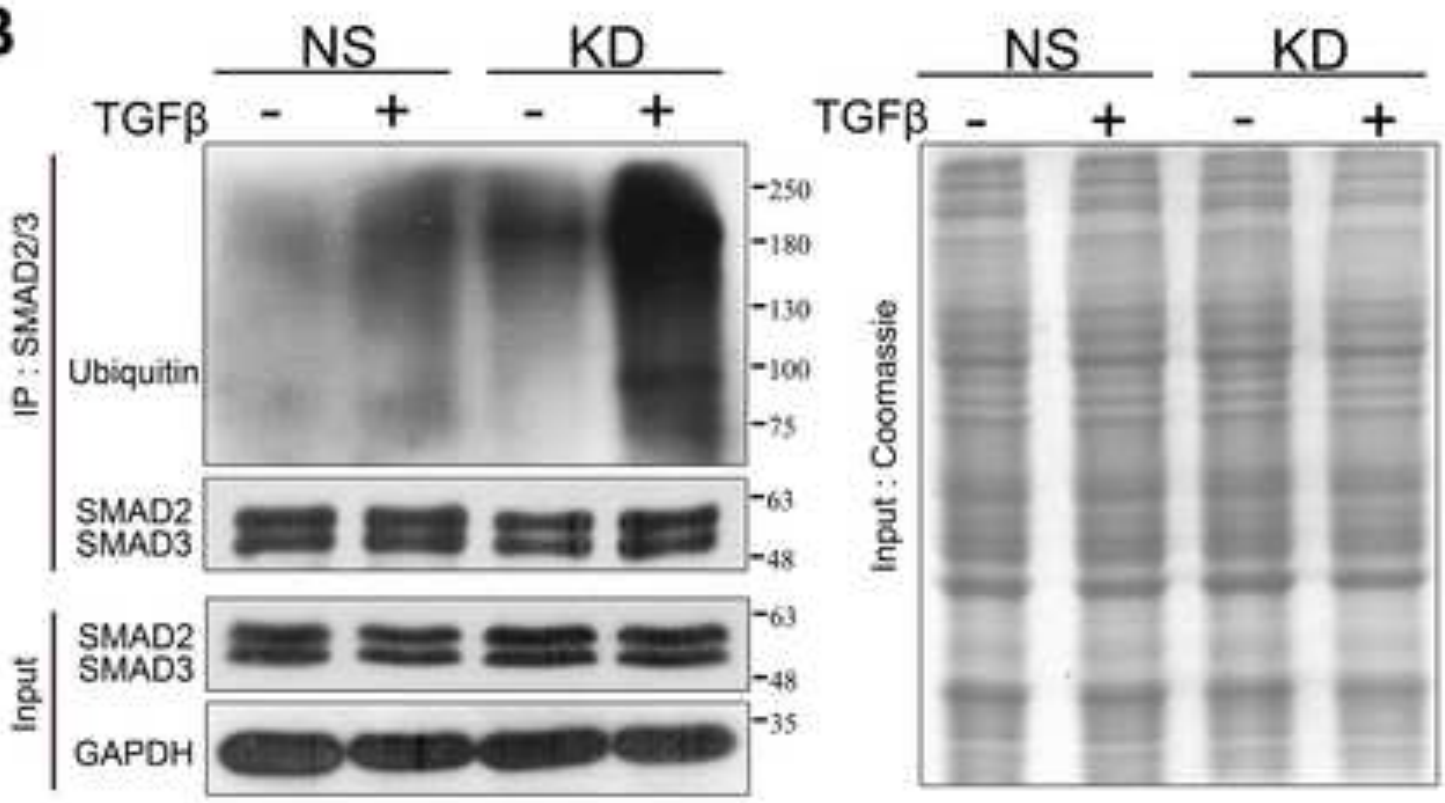

C
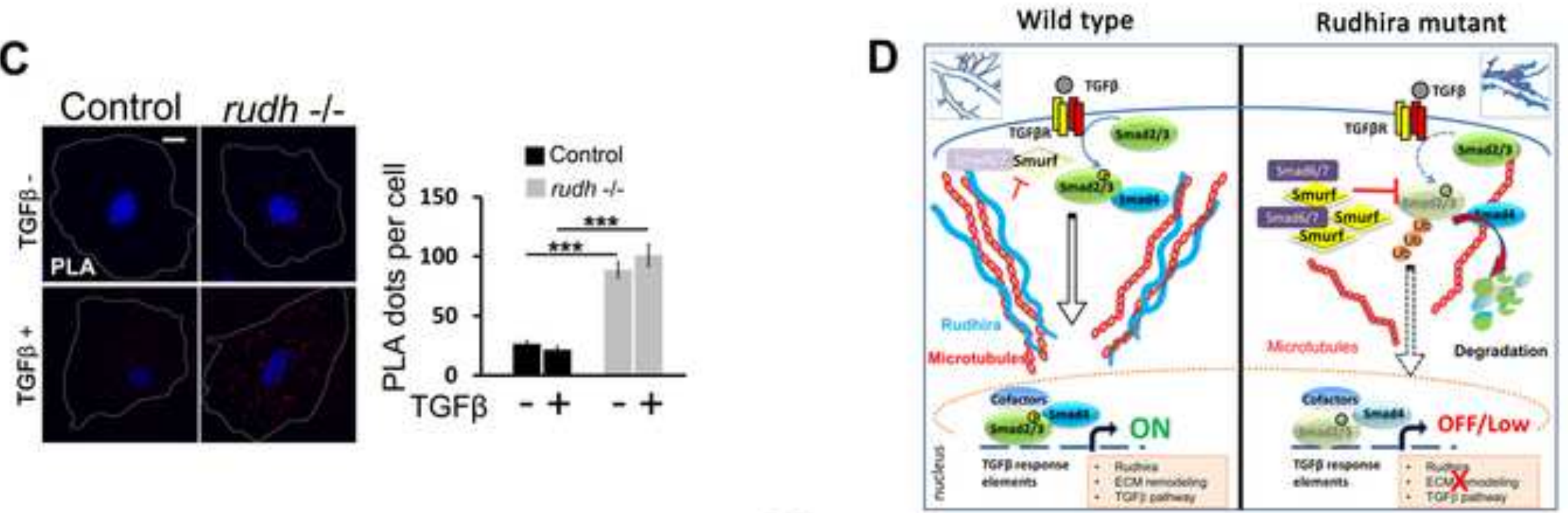

Figure 7

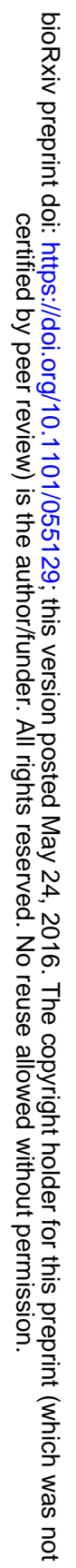

